\title{
Integrating multiple single-cell phenotypes links stress acclimation to prior life history in yeast
}

Andrew C. Bergen ${ }^{1}$, James Hose ${ }^{1}$, Megan McClean ${ }^{1,2,3}$, Audrey P. Gasch ${ }^{1,3,4}$

${ }^{1}$ Center for Genomic Science Innovation, University of Wisconsin-Madison, Madison WI 53706

${ }^{2}$ Department of Biomedical Engineering, University of Wisconsin-Madison, Madison WI 53706

${ }^{3}$ University of Wisconsin Carbone Cancer Center, University of Wisconsin School of Medicine and Public Health, Madison, Wisconsin

${ }^{4}$ Department of Medical Genetics, University of Wisconsin-Madison, Madison WI 53706

Running title: life-history states linked to stress response

Key words: stress response, fitness, heterogeneity, cell signaling, yeast

Acknowledgements: We thank Stephanie Geller, Taylor Scott, and Kieran Sweeney for help on microfluidics and microscopy, Michael Newton and Kirin Hong for statistical discussion, and members of the Gasch Lab for constructive comments. This work was supported by NSF grant 1715324 to APG.

\section{Abstract}

Stress defense and cell growth are inversely related in bulk culture analyses; however, these studies do not capture cellular heterogeneity, thus obscuring true phenotypic relationships. Here, we devised a microfluidics system to characterize multiple phenotypes in single yeast cells responding dynamically to stress. We simultaneously followed cell and colony growth, cell size and volume, and cell-cycle phase plus nuclear trans-localization of two transcription factors: stress-responsive activator Msn2 and repressor Dot6 that are co-regulated during stress. Coordinated activation reflects a 
systemic stress response, whereas decoupled behavior indicates factor-specific responses. We scored these features before, during, and after salt stress. Modeling of multi-cell phenotypes revealed surprising new information, including unexpected discordance between Msn2 and Dot6 behavior that revealed subpopulations of cells with distinct growth properties. Although past work connected Msn2 activation to growth rate, we instead found stronger correlations with Dot6 behavior. Post-stress growth rate could be partly predicted by integrating multiple cellular phenotypes, with higher accuracy than considering any single feature alone. Our results underscore that life-history experiences partially predict how cells will respond to stress.

\section{Introduction}

All organisms respond to stressful environments. Stress can come from external conditions including drugs and environmental shifts, or internal perturbations including mutation and disease. Thus, at the cellular level, organisms must be able to sense both external and internal signals to mount a proper response. Yet in both single- and multicelled organisms, there can be large variation in how individual cells respond to environmental stress, even among genetically identical cells in the same environment. For example, cell-to-cell variation in signaling and gene expression have been linked to differential survival of isogenic cancer cells responding to drugs (Lee et al. 2014; Paek et al. 2016; Shaffer et al. 2017; Inde and Dixon 2018). Similarly, cellular heterogeneity in bacterial growth and gene expression can produce variation in survival upon antibiotic treatment (Balaban et al. 2004; Keren et al. 2004). Understanding the nature of this variation could facilitate the modulation of stress survival, with therapeutic applications.

One marker of heterogeneity in stress responses is dynamic localization of stress-activated transcription factors. Several canonical factors, including p53 in mammalian cells (Purvis et al. 2012; Kracikova et al. 2013; Paek et al. 2016) and Msn2 and its paralog Msn4 in fungi (Görner et al. 1998), reside in the cytosol in the absence 
of stress but rapidly translocate to the nucleus upon activation. These and other stressactivated factors can vary substantially in their responsiveness, in ways that can impact cellular outputs including gene-expression. For example, Msn2 localization dynamics differ depending on the nature of the stress (Hao and O'Shea 2012; Petrenko et al. 2013; Granados et al. 2018), and these differences impart distinct effects on different target genes (Hao and O'Shea 2012; Hansen and O'Shea 2013; Stewart-Ornstein et al. 2013; Hansen and O'Shea 2015a; Hansen and O'Shea 2015b; Hansen and O'Shea 2016; Hansen and Zechner 2021). Genes with highly responsive promoters can be induced even with brief pulses of nuclear Msn2, whereas genes with weaker promoters require prolonged Msn2 activation (Hansen and O'Shea 2013; Hansen and O'Shea 2015a; Hansen and O'Shea 2015b; Hansen and O'Shea 2016). Similarly, different patterns of p53 localization dynamics can alter expression of different gene sets, and these differences correlate with differences in stress survival (Purvis et al. 2012). Several studies have observed substantial cell-to-cell heterogeneity in nuclear localization dynamics of these factors (Cai et al. 2008; Cheong et al. 2011; Purvis and Lahav 2013; Lin et al. 2015; AkhavanAghdam et al. 2016; Gasch et al. 2017; Granados et al. 2018; Li et al. 2018); however, the causes and functional effects of this variation remain poorly understood.

Cell-to-cell variation in transcription factor localization dynamics could arise for several reasons. Changes in the state of a single transcription factor may alter its localization independent of the cellular system (defined as factor-specific variation). In contrast, activity-state changes in the upstream signaling networks or cellular system itself could produce coordinated activation of the stress response (referred to as 
systemic variation). Distinguishing between local versus systemic variation has been difficult, since most studies to date have followed only single transcription factors. We recently developed strains in which two differentially tagged transcription factors regulated by the same signaling network are expressed in the same yeast cell. Msn2 activator fused to mCherry is co-expressed with the transcriptional repressor Dot6 fused to GFP. Both factors help to coordinate the yeast environmental stress response (Gasch et al. 2000; Causton et al. 2001): whereas Msn2 activates defense genes that are induced in the ESR (iESR genes), Dot6 represses growth-promoting genes involved in ribosome biogenesis that are correspondingly repressed in the ESR during stress (rESR genes) (Lippman and Broach 2009; Bergenholm et al. 2018). Both factors are controlled by the Protein Kinase A (PKA) and mTOR pathways, which are generally associated with promoting growth (Figure 1A): PKA/TOR-dependent phosphorylation of Msn2 and Dot6 maintains the factors in cytosol, whereas Msn2 and Dot6 dephosphorylation after PKA/TOR inhibition leads to their nuclear localization. (Görner et al. 1998; Smith et al. 1998; Lippman and Broach 2009). Thus, we expect the two factors to be coordinated in their localization when the stress response is activated systemically but discordant in response to factor-specific differences in regulation.

The challenges in distinguishing factor-specific versus systemic variation have obscured how systemic activation of the stress response relates to other physiological responses. One important factor is growth rate. Growth rate and stress tolerance are competing interests in the cell and are often antagonistically regulated: fast growing cells tend to be the most susceptible to stress and toxins, whereas slow growing or quiescent cells generally survive extreme conditions (Balaban et al. 2004; Lu et al. 
2009; Zakrzewska et al. 2011; Levy et al. 2012). Part of this antagonistic correlation is thought to be controlled, at least under specific situations, by the RAS-PKA pathway, which promotes growth and suppresses the stress response (Smith et al. 1998; Gasch et al. 2000; Zaman et al. 2008; Zaman et al. 2009). Li et al. (2018) used a microfluidics system to show that slower growing cells in an isogenic culture showed lower levels of the PKA allosteric activator CAMP and that artificial activation of PKA diminished the slow growing population (Li et al. 2018). They further showed a slight but statistically significant negative correlation between Msn2 nuclear localization and growth of microcolony size over the subsequent 10 hours in the absence of stress. This suggests that activation of Msn2 is coupled to reduced growth rate, a theory put forward and debated in other bulk-culture studies (Regenberg et al. 2006; Castrillo et al. 2007; Brauer et al. 2008; Ho et al. 2018). The inability to distinguish between factor-specific variation and systemic activation of the stress response likely obscures the true relationship with growth.

Here we monitored dynamic localization changes of both Msn2 and Dot6 in the same yeast cells, along with a panel of other single-cell measurements, to dissect local and systemic variation and illuminate the relationship between ESR activation and growth rate. We optimized a microfluidics system that can monitor single-cell localization levels and dynamics of both Msn2-mCherry and Dot6-GFP along with single-cell and colony growth rates, size, shape, cell-cycle phase and volume changes before and after an acute dose of sodium chloride $(\mathrm{NaCl})$ as a model stressor. Our results revealed several insights, including surprising levels of discordance in Msn2 and Dot6 activation that suggest previously unknown regulatory distinctions and uncover 
subpopulations of cells. We developed a multi-factorial model explaining cell growth rate after stress acclimation to demonstrate that stress acclimation is partly predictable based on prior life history states. Our work highlights that quantifying and integrating multiple phenotypes, including the behavior of multiple transcription factors in the same cells, increases predictive power and contributes a deeper understanding of the causes and consequences of heterogeneous stress responses.

Results

We optimized a microfluidics system that could measure nuclear localization dynamics as well as one- and two-cell colony growth rates before and after exposure to 0.7 $\mathrm{M} \mathrm{NaCl}$ (Figure 1B and Methods). Using this system, we characterized the variation in cell responses for 72 min before and 144 min after exposure to $\mathrm{NaCl}$, which induces ionic and osmotic stress, in biological triplicates done on separate days. This time frame captures phenotypic variation in cells growing in the absence of stress, during the acute stress-response phase (from 0 to 54 minutes after osmotic stress), and over later timepoints as cells acclimate to continuous $\mathrm{NaCl}$. Microscopy imaging and analysis reports on Msn2-mCherry and Dot6-GFP nuclear localization dynamics in the same cells (Figure 1C). We used MATLAB scripts to identify nuclear translocation events, which we refer to as "peaks" in the traces (see Methods). We also measured cell and colony growth phenotypes, including colony size, colony growth rates (defined by increase in pixel number of masked colony area) both before and after stress, and volume lost upon $\mathrm{NaCl}$ stress (Figure 1D and Methods). To insure accurate measures of growth, we limited our analysis to colonies of only one or two cells at the beginning of 
the time series and to cells that passed several quality-control filters (see Methods). In total, we analyzed 221 cells passing these filters, collected from the three independent biological replicates.

\section{Figure 1}

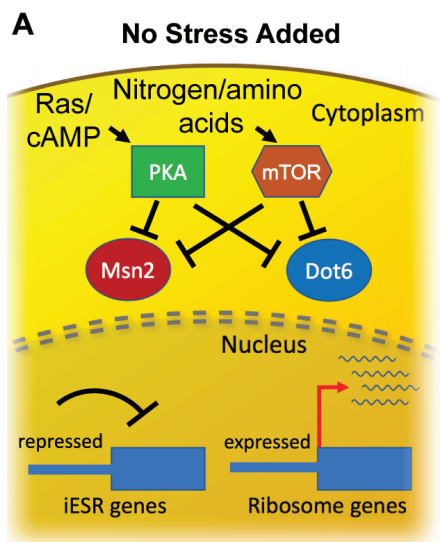

C

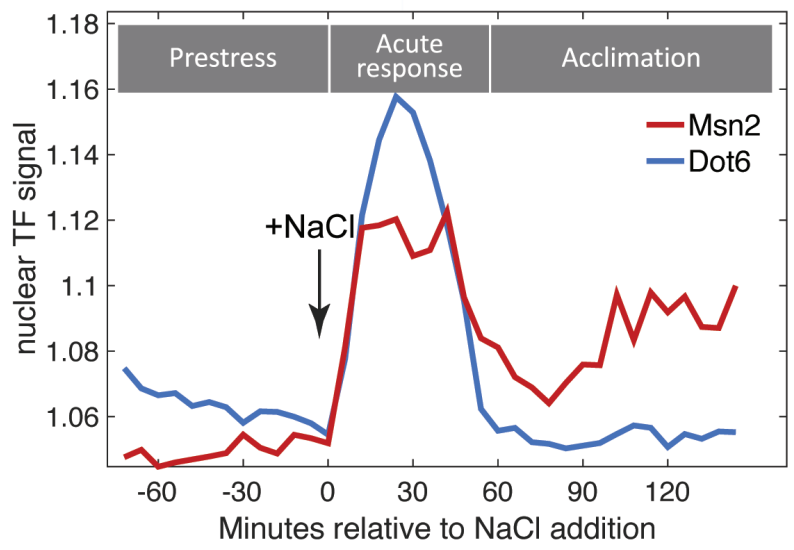

B

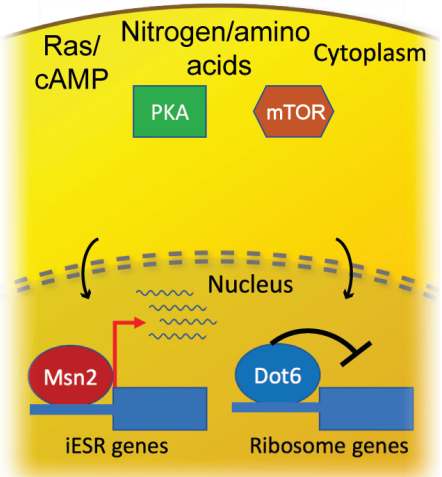

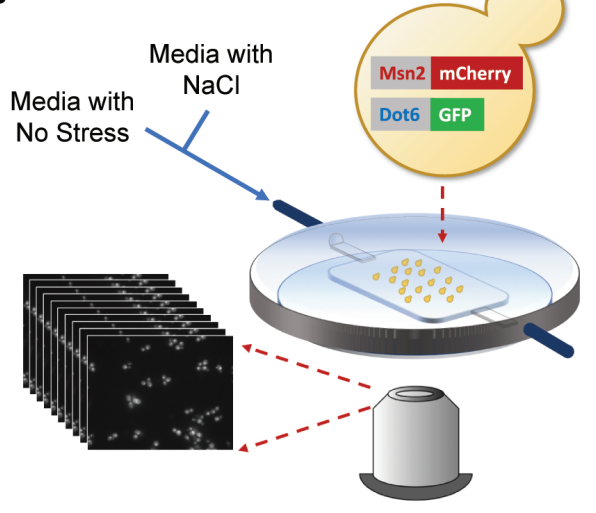

D

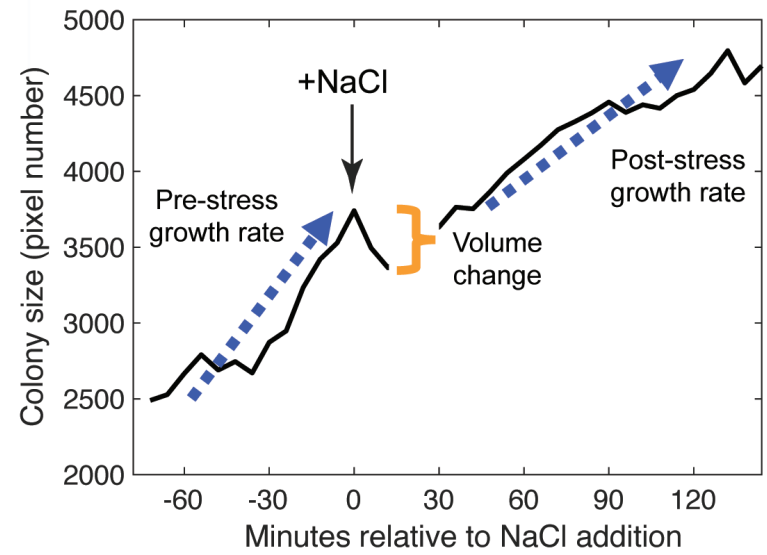

Figure 1. Experimental approach. A. Schematic of Msn2 and Dot6 localization in the absence (left) and presence (right) of stress. B. Diagram of microfluidic device used for time-lapse microscopy. C. Representative nuclear localization scores (see Methods) for pre-stress growth, the acute-stress response, and the acclimation phase. D. Cell or twocell colony size was estimated by the number of pixels within the mask for each colony, and growth rates were calculated based on the regressions of those points during the pre- or post-stress phases. Cell volume change was calculated as the difference in pixel number before and after stress. 
This system captured variation in all of the features measured. As expected based on previous studies (Levy et al. 2012; Fehrmann et al. 2013; Crane et al. 2014; Li et al. 2018; Jin et al. 2019), there was substantial variation in cellular growth rates before $\mathrm{NaCl}$ addition, confirming that cells vary considerably in their growth properties in the absence of stress (Figure 2A). Most colonies reduced their growth rate in response to $\mathrm{NaCl}$ stress, but once again there was substantial variation: some cells showed dramatic growth reduction upon $\mathrm{NaCl}$ whereas others showed little to no change (Figure 2B). There were even individual colonies that accelerated growth after stress: 11 of 14 of these cells showed a small bud immediately before, or at the time of salt exposure, suggesting a cell cycle connection (Figure 2B). NaCl-induced osmotic pressure is expected to produce rapid water loss before cells acclimate, and indeed most cells lost volume immediately after stress despite substantial variation in volume change (Figure 2C). Together, these results highlight the extensive cell-to-cell variation in behavior that is not identified in bulk measures of culture growth.

\section{Figure 2}
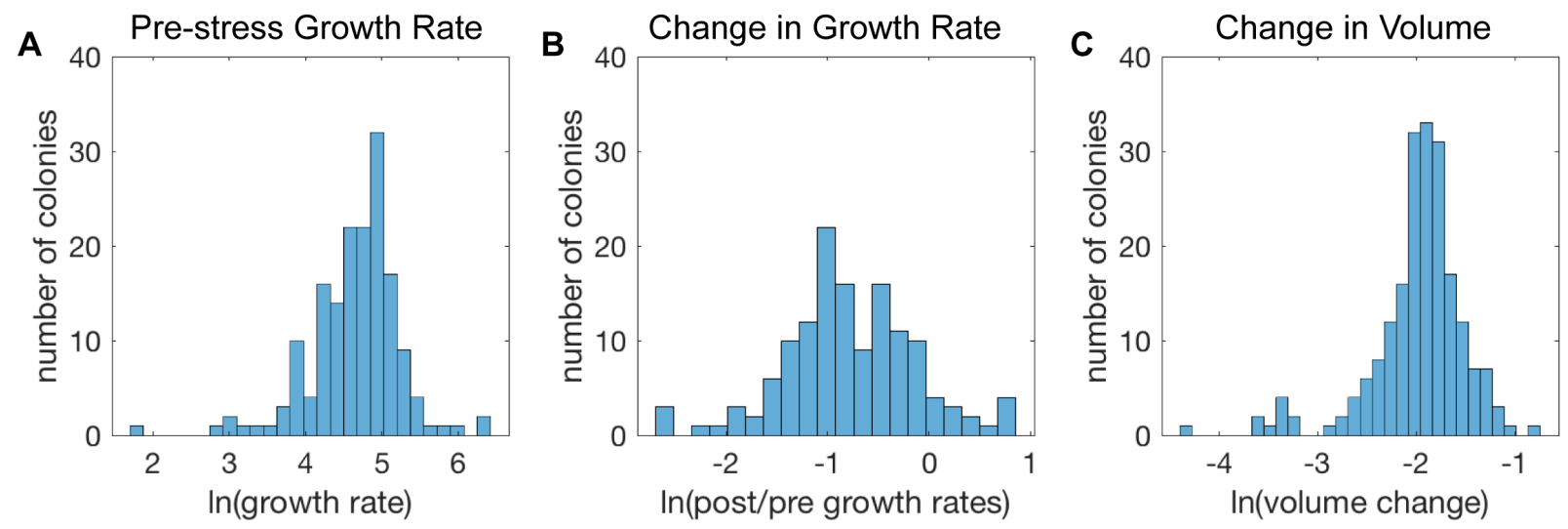

Figure 2. Cell-to-cell heterogeneity in the $\mathrm{NaCl}$ stress response. A-C. Shown are the distributions of the natural log of $\mathbf{A}$. colony growth rates before stress, $\mathbf{B}$. the change in growth rate after $\mathrm{NaCl}$ stress compared to before stress, and $\mathbf{C}$. the maximum 
change in cell volume during the acute-stress response versus during the pre-stress phase.

\section{Msn2 and Dot6 nuclear localization shows only partial coordination}

We next investigated co-variation in Msn2-mCherry and Dot6-GFP localization dynamics, before and as cells responded to $\mathrm{NaCl}$. Both factors showed sporadic activation in unstressed cells, with brief and typically low levels of nuclear translocation (Figure $3 A$ ). In roughly $21 \%$ of cells, pre-stress peaks of Dot6 and Msn2 were temporally coordinated with the other factor (Figure 3B), which is significantly above chance ( $p<<0.0001$, permutation analysis, see Methods) and suggests systemic activation of the stress response. This reveals both coordinated and independent fluctuations in Msn2 and Dot6 activation in the absence of stress, consistent with our prior results (Gasch et al. 2017). In the vast majority of cells, $\mathrm{NaCl}$ provoked a dramatic and coordinated increase in nuclear localization of both Msn2 and Dot6 (acute phase). However, after stress Msn2 and Dot6 behavior deviated: whereas few cells showed post-stress Dot6 nuclear translocation, many cells showed asynchronous pulses of Msn2 (Figure 3C), consistent with prior work (Petrenko et al. 2013). This was surprising, since we expected that Msn2 and Dot6 would be highly correlated during and immediately after $\mathrm{NaCl}$ treatment.

Plotting the aggregate and standard deviation of nuclear localization over all cells highlighted the different behaviors of the two factors (Figure 3D-F). The population deviation for both transcription factors was similar during the pre-stress phase, consistent with the significant co-variation of the two factors. However, during the acute 


\section{Figure 3}

A

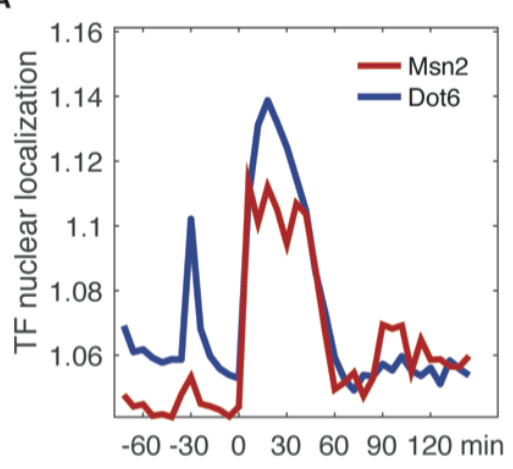

D 1.16

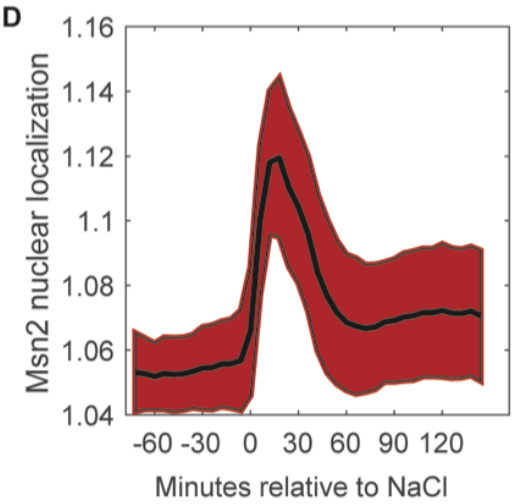

B
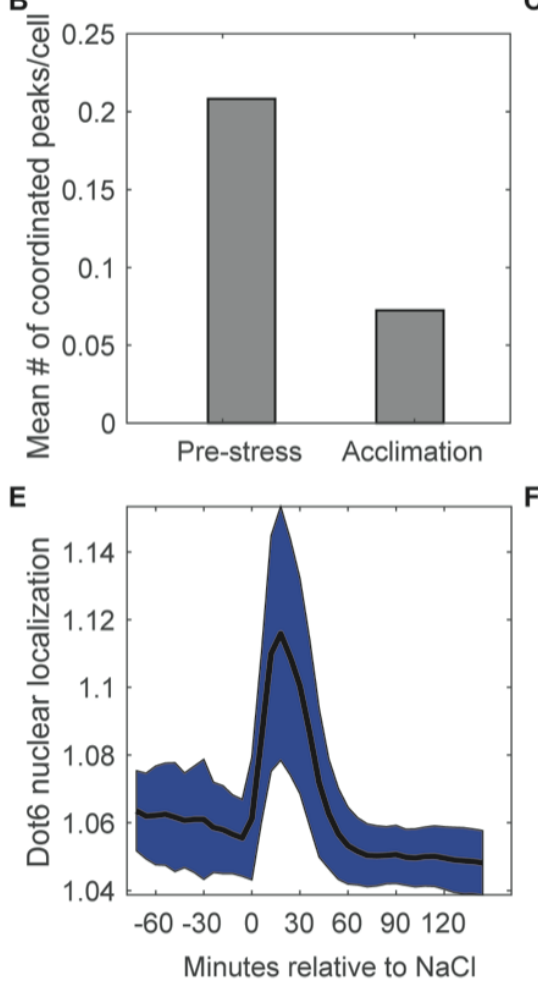

C
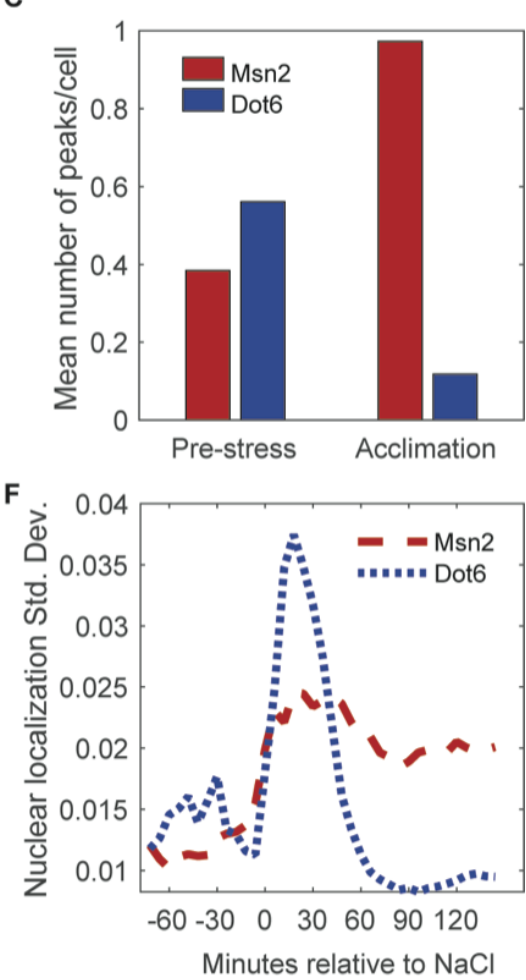

Figure 3. Nuclear translocation dynamics of Msn2 and Dot6 are more coordinated before stress. A. Representative traces of Msn2 and Dot6 in the same cell. B. The average number of coordinated peaks for Msn2 and Dot6, i.e. those called within 6 minutes (1 timepoint) of each other. C. The average number of called nuclear localization peaks per cell for Msn2 (red) and Dot6 (blue) during pre-stress and acclimation phases. D-E The average (black line) +/- one standard deviation (colored spread) of Msn2 (D) and Dot6 (E) nuclear localization during the time course. F. Trace of the standard deviation of nuclear localization over the time course for Msn2 (red) and Dot6 (blue).

response immediately after $\mathrm{NaCl}$ exposure, the standard deviation of Dot6 was much wider than that of Msn2, indicating more variable Dot6 responses across the cells. In contrast, the standard deviation of Dot6 was very low during the acclimation phase but remained high for Msn2 (Figure 3F). These results suggested that, even during a 
coordinated stress response, there are substantial differences in heterogeneity for Msn2 versus Dot6.

In the course of this analysis, we realized another key difference between Msn2 and Dot6: the timing of Dot6 nuclear pulses was often correlated between unstressed cells in the same two-cell colony, indicated by co-occurring peaks in colonies (Table S1). Permutation tests showed that this was significant compared to random chance (p $=9.3 e-4$, see Methods). In contrast, the number of co-occurring Msn2 peaks in cells in the same two-cell colony was not significantly different from random. Since these cells are in the same local environment and have a shared life history in that one cell is the daughter of the other, it suggests that some feature of Dot6 regulation is predictable but separable from Msn2 behavior (see Discussion).

\section{Reproducible differences in Msn2 versus Dot6 activation reveal subpopulations of cells}

Comparisons of Msn2 and Dot6 nuclear localization patterns indicated different localization dynamics across cells, raising the possibility of distinct cell subpopulations. To investigate, we used Gaussian finite mixture modelling (Scrucca et al. 2016) of the population-normalized Msn2 and Dot6 nuclear localization traces to identify 11 populations or 'clusters' of cells with distinguishable localization patterns (Figure 4, see Methods). Most clusters captured cells from all three biological replicates, with the exception of cell Cluster 9 and several small clusters that were enriched for cells from one replicate (Table S2). Thus, most of the cell groupings represent reproducible subpopulations with different stress-responsive behaviors. 
Figure 4

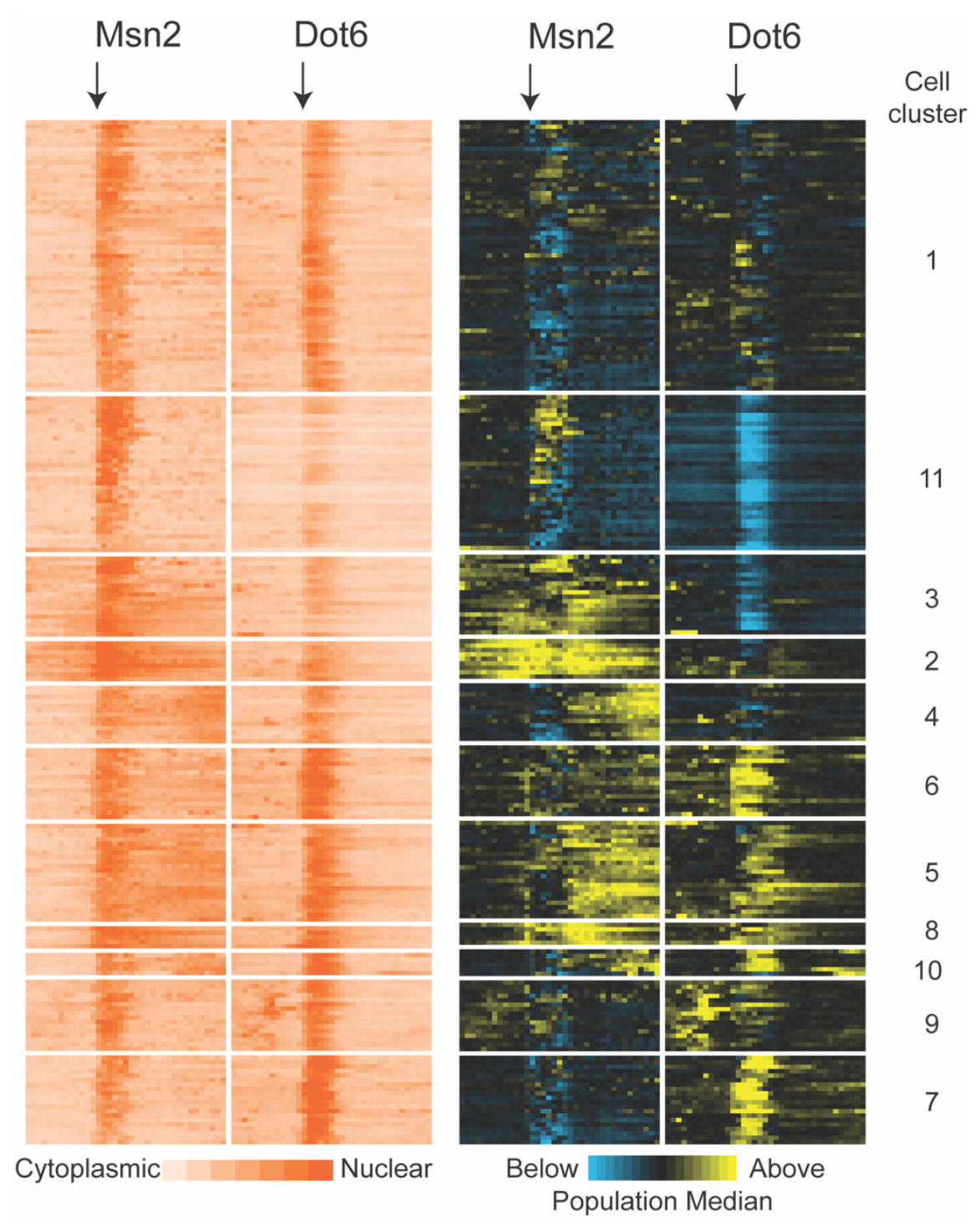

Figure 4. Subpopulations of cells show distinct Msn2 and Dot6 translocation dynamics. 221 cells passing quality control metrics were partitioned into sub clusters based on their population-centered nuclear translocation dynamics shown on the right. Each row represents a cell and each column in a block represents a single timepoint; time of $\mathrm{NaCl}$ addition is indicated with an arrow. Data on the left show the $\log _{2}$ ratio of nuclear versus total Msn2 (left) or Dot6 (right) according to the orange-scale key, see Methods. Data on the right show the same data normalized to the population median at each timepoint: yellow values indicate higher-than-median nuclear localization and blue indicates lower-than-median nuclear localization. Cell clusters identified by the package mclust are labeled to the right. 
The subpopulations were differentiated by a combination of transcription-factor phenotypes. One distinguishing feature was the level of Dot6 activation during the acute-stress phase. Cluster 11 was characterized by lower than population-median magnitude of acute-stress Dot6 nuclear translocation, whereas cells in Clusters 6 and 7 showed higher-than-average Dot6 response. These results are consistent with the wider variance of Dot6 nuclear translocation levels during the acute phase (Figure 2D-F). A second distinguishing feature was the level of nuclear Msn2 and Dot6 before stress. Cluster 11 cells showed low levels of Dot6 before stress, whereas cells in Clusters 9 and 6 displayed higher-than-median nuclear Msn2 and Dot6 during this phase. Finally, the behavior of Msn2 during the post-stress acclimation phase was significantly different across subpopulations. Whereas Clusters 11 and to some extent 7 showed low levels of post-stress Msn2 nuclear localization, cells in multiple clusters showed high levels and/or pulsatile nuclear Msn2 as cells acclimated. We noticed that cells in Clusters 2 and 3 showed elevated levels of Msn2 that persisted over time compared to other cells. Closer inspection of the microscopy images suggested that some of the signal may not reflect nuclear translocation but instead was likely vacuolar signal (see more below). As mentioned above, the variation in nuclear localization dynamics captured within these clusters occurred in all three biological replicates (See Table S2), indicating reproducible distinctions in transcription factor behavior. Together, this analysis revealed important differences in cellular behavior across the phases of the $\mathrm{NaCl}$ response that are obscured by aggregate analysis of all cells in the population.

\section{Cell subpopulations show different relationships with cell growth}


Are subpopulations of cells identified above biologically meaningful? We turned to the other cellular measurements to look for co-variates in cellular behavior that reflect on higher-order relationships. We tested each of the cell subpopulations for statistically significant differences in pre-stress growth rate, post-stress growth rate, starting size, volume change, and cell-cycle phase at the time of $\mathrm{NaCl}$ exposure (inferred by visual inspection of bud size and nucleus location in the cell, see Methods). We found no significant correlations with cell volume changes or cell-cycle phase (although there was a minor signal for cell cycle, Figure S1). This is consistent with the lack of strong connection between cell-cycle phase and stress response found in several other studies (Paek et al. 2016; Gasch et al. 2017; Bagamery et al. 2020). In contrast, several clusters showed significant differences in growth rates.

Overall, there was a positive correlation between pre-stress colony growth rate compared to post-stress growth rate (Figure 5A); however, the association was different for subpopulations of cells. Cells in Cluster 11, which were characterized by below-average Dot6 response before and during stress, showed slower growth rates before being exposed to stress and after $\mathrm{NaCl}$ treatment (Figure 5B-C), and the slower growth was consistent across biological replicates $(p<0.02$, Wilcoxon rank sum for each replicate analyzed separately). In contrast, cells in Cluster 7 showed higher than average recovery growth rates - these cells were characterized by larger-than-average Dot6 nuclear localization responses and somewhat below-average nuclear translocation of Msn2 during the acute-response phase. The relationships between post-stress growth rate and Dot6 response during the acute phase raised the possibility that this factor's activation is more closely tied to growth rate than Msn2, even when both factors 
are activated in a systemic response. Interestingly, cells in Cluster 2 that had unusually high (and potentially vacuolar) red-channel fluorescence before stress displayed very slow growth recovery after stress, demonstrating the biological validity of the subpopulation and raising the possibility of poor stress acclimation in these cells. (We note that cells with apparent vacuolar signal were excluded from subsequent analyses).

\section{Figure 5}

A

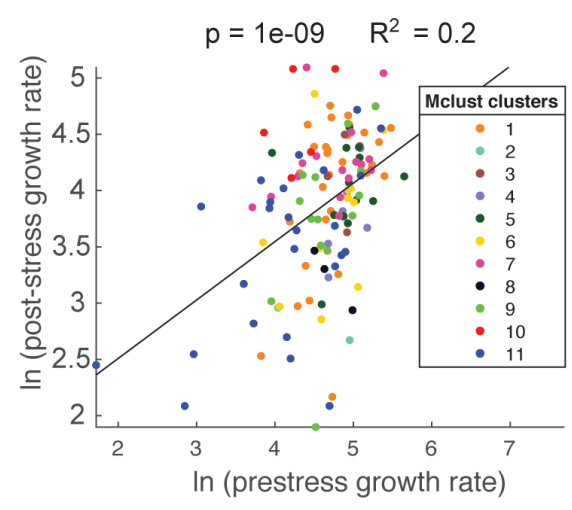

B

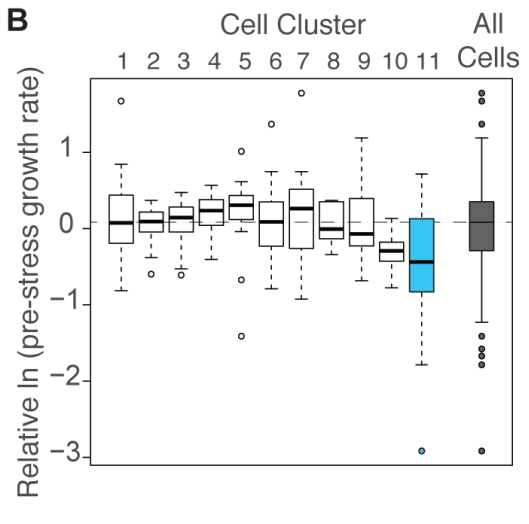

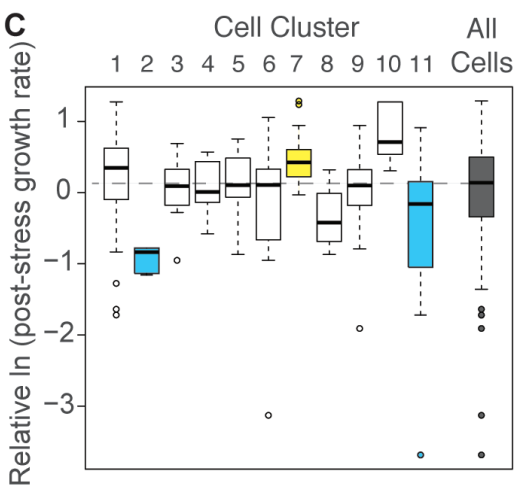

Figure 5. Cell subpopulations display different growth rates before and after stress. A. Correlation between the natural log of pre- and post-stress growth rates for each cell, colored according to its cell cluster in Figure 4. B-C. Distribution of mediancentered growth rates before (B) and after (C) $\mathrm{NaCl}$ addition, for cell clusters shown in Figure 4. Boxes are colored yellow or blue if the distribution was significantly higher or lower, respectively, from all other cells in the analysis (Wilcoxon Rank Sum test, FDR < 0.022). Dashed line indicates the median of all cells analyzed.

\section{Combining multiple characteristics increases the predictive power to explain}

growth

The above results hinted that how well cells acclimate to $\mathrm{NaCl}$ stress, as indicated by post-stress growth rate, may be predicted by cellular responses both 
before and during the stress response. Based on the work of (Li et al. 2018), we expected a negative correlation between Msn2 nuclear localization and growth rate. While there was no correlation with pre-stress growth rate $(p=0.65)$, we indeed observed a negative correlation between pre-stress Msn2 activation (taken as the area under the nuclear-localization curve (AUC) for pre-stress timepoints) and post-stress growth rate (Figure 6A). However, the correlation explained only $3 \%$ of the variance $(p$ $=0.016$, linear regression), indicating that the pre-stress behavior of Msn2 has little power to predict growth rate.

We next investigated other features that could explain differences in post-stress growth rate (Figure 6B). Pairwise correlations revealed that some individual features, such as the magnitude of Dot6 acute-stress response, correlated well with post-stress growth rate but others did not (Figure S2). However, the most impactful single factor, pre-stress growth rate, explained only $20 \%$ of the variance in post-stress growth rate (Table S3).

We next asked if combing factors into a single multiple linear model could explain more of the variance in growth. We considered multiple metrics for summarizing prestress nuclear localization, including AUC (which is a measure of the overall nuclear abundance) and the sum of called translocation peak heights (which is influenced by the magnitude and frequency of pre-stress pulses), along with acute-stress translocation 
Figure 6

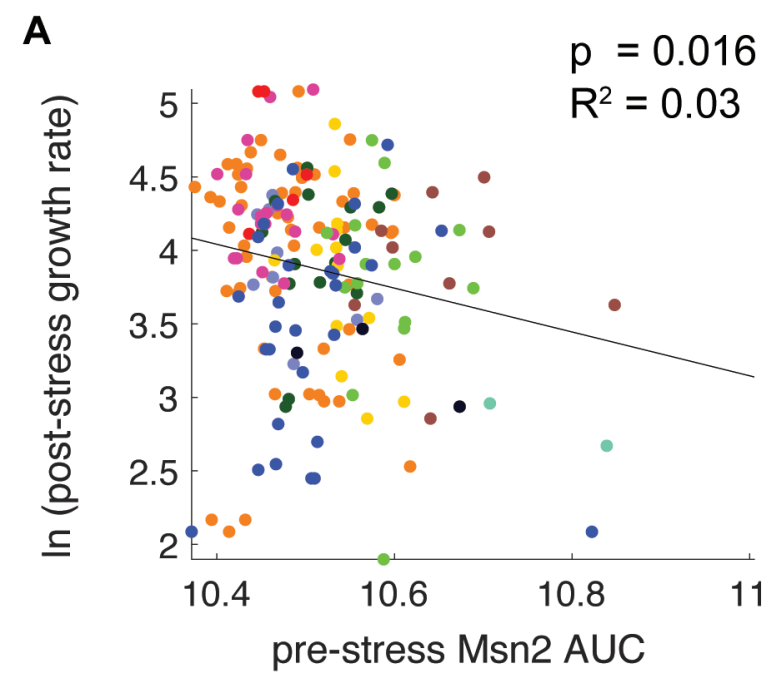

B

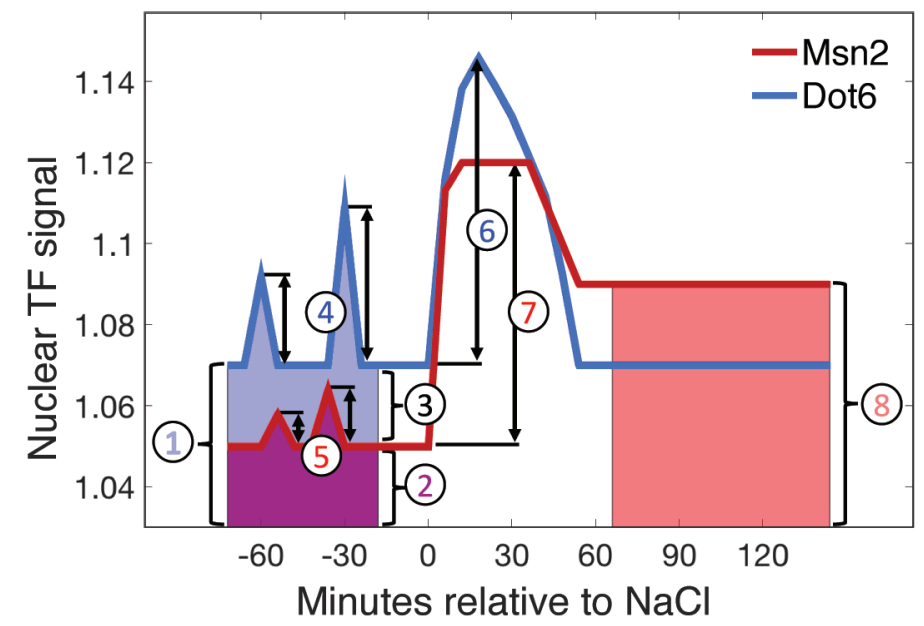

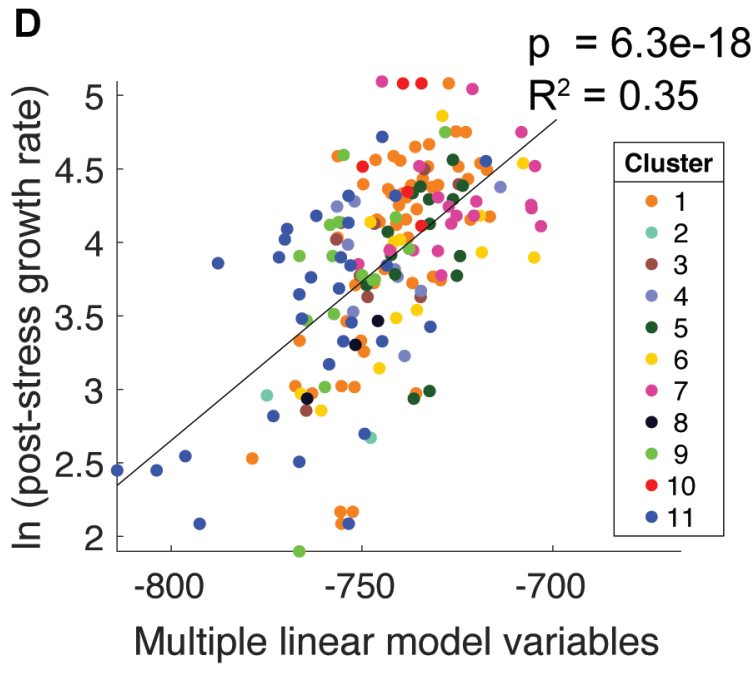

C

Tested Model Parameters

1) Pre-stress Dot6 AUC ***

2) Pre-stress Msn2 AUC

3) Pre-stress Msn2 AUC vs. Dot6 AUC

4) Sum pre-stress Dot6 peak heights

5) Sum pre-stress Msn2 peak heights ***

6) Acute-stress Dot6 peak change ***

7) Acute-stress Msn2 peak height

8) Acclimation-phase Msn2 AUC

9) Pre-stress growth rate ***

10) Cell cycle stage

11) Cell size

Figure 6. A multi-factor model best explains variation in post-stress growth rate. A. Regression of the In(post-stress growth rate) against the pre-stress Area Under the Curve (AUC) of Msn2 localization. Each point represents a different cell, color coded by its subpopulation indicated in panel $D$. The $p$-value and $R^{2}$ are shown on the plot. $B$. Pictorial representation of the nuclear localization measurements used in the multifactor linear regression model. C. Factors considered in the multi-factor linear regression model; those with significant contributions are highlighted with ${ }^{* * *}$. D. The variance in In(post-stress growth rate) explained by the multi-factor linear regression model. P-value and $\mathrm{R}^{2}$ are shown a the top of the plot and cell subcluster is indicated according to the key.

peak height and AUC during the acclimation phase. The model also incorporated other cell features including pre-stress growth rate, cell-cycle phase at the time of $\mathrm{NaCl}$ 
exposure, and cell size factors (See 'Model 1' in Table S3 for all parameters used).

Factors that did not contribute significantly (adjusted $p>0.05$ ) were progressively removed until the variance explain decreased (Table S3). The final regression identified 4 factors that contributed significantly to explain post-stress growth rate ('Model 3' in Table S3): pre-stress AUC of Dot6 nuclear localization, the sum of pre-stress Msn2 peak heights, the pre-stress growth rate of the cells, and the magnitude of Dot6 nuclear localization change immediately after $\mathrm{NaCl}$. Together, these factors - all but one of which represent pre-stress cellular phenotypes - explained $35 \%$ of the variance in poststress growth rate (Figure 6C), nearly doubling the explanatory power of any single feature alone (Figure 6D, S2). Surprisingly, the sum of pre-stress Msn2 peak heights and pre-stress AUC of Dot6 nuclear localization both contributed significantly to the multi-factor model but were not predictive when considered individually (Figure S2). This suggests that these measures of transcription-factor nuclear localization can explain residual variance even though they explain a small portion of the data when considered alone.

\section{Dot6 responses to stress are influenced by pre-stress life history states}

We were surprised to find that the magnitude of the Dot6 nuclear translocation during the acute stress response was among the most predictive of post-stress growth rate, whereas the Msn2 behavior contributed little explanatory power. We therefore sought factors that could explain the variation in Dot6 peak height during the acute response. We measured co-variation of Dot6 acute-stress peak height and a panel of features and realized several relationships. Although the magnitude of Msn2 nuclear 


\section{Figure 7}

A

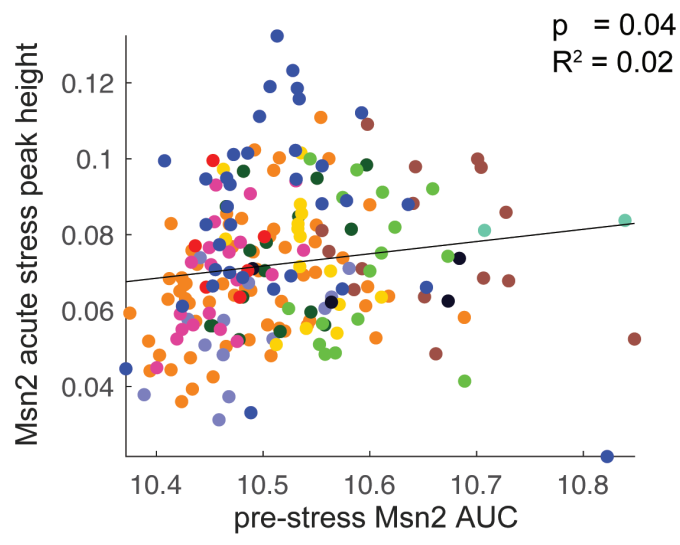

C

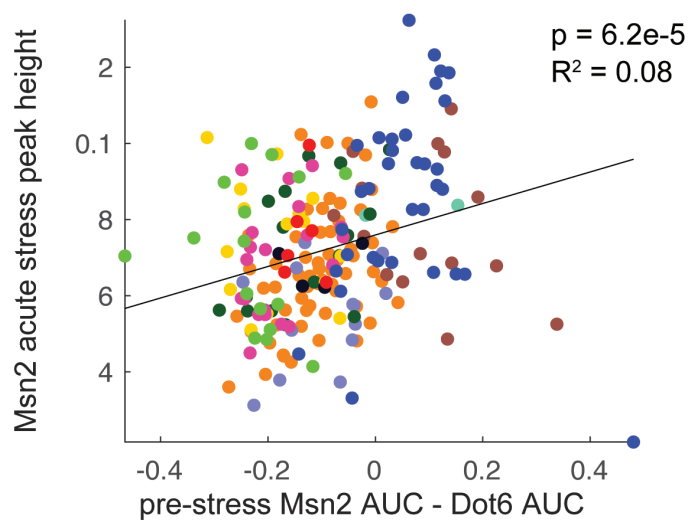

E

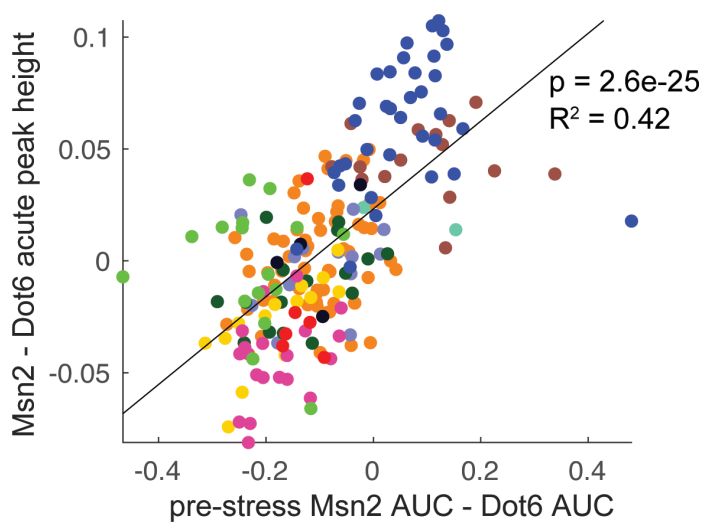

B

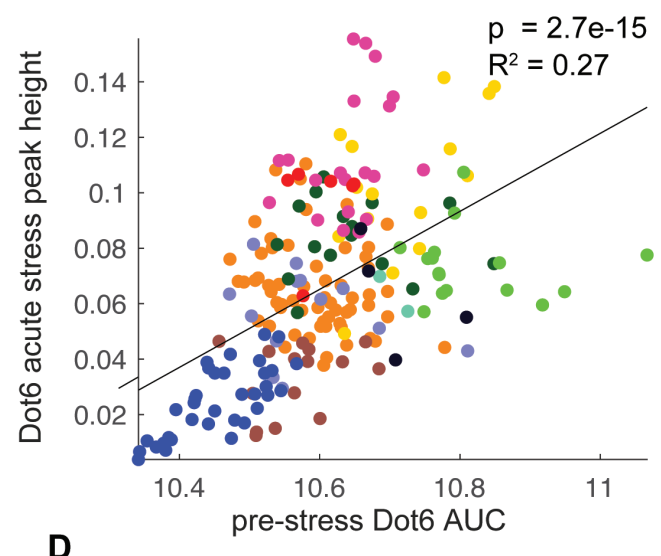

D
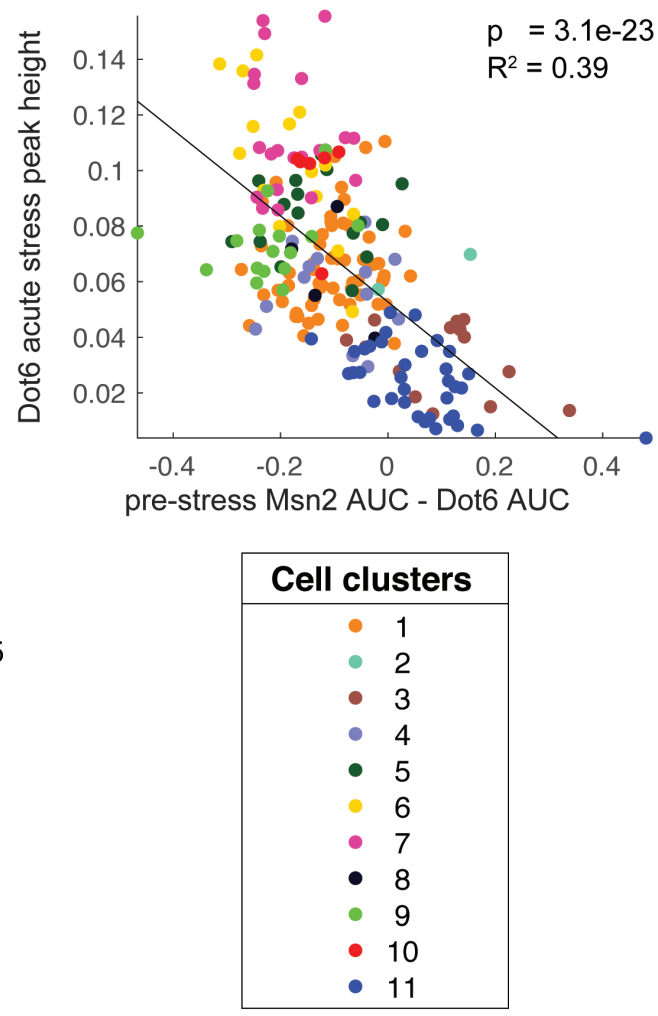

Figure 7. Pre-stress cell characteristics explain the Dot6 stress response. Acute stress peak heights and pre-stress area under the curve (AUC) are as defined in Figure $6 \mathrm{~B}$, and cell points are colored according to their cell cluster from Figure 4 as shown in the key. A-B. Acute-stress peak height plotted against pre-stress AUC for Msn2 (A) or Dot6 (B). C-D. Acute-stress peak height for Msn2 (A) or Dot6 (B) plotted against the 
difference in pre-stress AUC. E. The difference in pre-stress Msn2 and Dot6 nuclear localization best explains the difference in acute-stress peak height for Msn2 and Dot6. $P$-values and $R^{2}$ of the fit are shown on each plot. All $p$-values were significant at a Benjamini-Hochberg corrected false discovery rate of 0.05 .

localization upon $\mathrm{NaCl}$ stress was not strongly influenced by pre-stress Msn2 activation (Figure 7A), we did observe a significant positive relationship between Dot6 acutestress peak height and Dot6 AUC in pre-stressed cells - cells with more pre-stress activation of Dot6 had a larger Dot6 response to $\mathrm{NaCl}$ (Figure 7B). The trend was recapitulated in a strain in which the fluorescent tags were swapped (Msn2-GFP, Dot6mCherry, see Figure S3), indicating that it is not an artifact of the tags. It is important to note that the relationship was influenced by cells in cell Cluster 11 , since removing those cells yielded a significant correlation but with reduced the variance explained (from $29 \%$ to $6 \%$, see Figure S4B).

We noticed when considering cell clusters in Figure 4 that many subpopulations showed inverse trends in pre-stress Msn2 versus Dot6 activation. Several clusters that had higher nuclear levels of Dot6 before stress had lower levels of Msn2, and vice versa. This was especially surprising, since Msn2 and Dot6 are thought to be coordinately activated to mount the yeast ESR. We therefore wondered if the relative activation of Dot6 versus Msn2 before stress was any indication of different cellular states. In fact, the relative difference in Msn2 AUC versus Dot6 AUC before stress was more predictive of the acute-stress nuclear translocation peak of both Msn2 and Dot6 than considering either pre-stress factor alone (Figure 7C-D). To ensure that this was not an artifact of the fluorescent tags, we repeated the analysis in a strain in which the tags were swapped (Msn2-GFP, Dot6-mCherry). The trend was repeated for Msn2 but not Dot6 (perhaps owing to the unusually high level of variance explained in this 
experiment by pre-stress Dot6 AUC alone, Figure S3). For both strains, the variance explained for acute-stress response was in fact highest when assessing the relative peak heights of Msn2 versus Dot6 during the acute- $\mathrm{NaCl}$ response compared to the relative Msn2 and Dot6 activation during the pre-stress treatment (Figure 7E and S3E). Although we cannot exclude the possibility that the improved fit is a technical artifact, it is notable that the fits with other features, including growth rate modeling, was not improved by considering the relative pre-stress activation of Msn2 versus Dot6 compared to considering Dot6 pre-stress behavior alone. This raises the possibility that the differences in Msn2 and Dot6 behavior differentiate cells in a way that is predictive of distinct subsequent stress responses (see Discussion).

\section{Discussion}

Scoring multiple regulators in the same cell can provide additional information on a cellular response, as done in a handful of prior studies in yeast (Lin et al. 2015; AkhavanAghdam et al. 2016). Here, by following dynamic activation of two different transcription factors thought to be co-regulated during stress responses, in conjunction with other cellular features including growth rate, cell size, and cell cycle stage, we uncovered surprising new information about the yeast stress response and the interplay between growth versus stress defense. Characterizing the behavior of both Msn2 and Dot6 revealed reproducible subpopulations of cells that are not distinguished by following either factor alone. Several subpopulations showed differences in cellular growth rate, either before or after stress, indicating that the cell classifications are biologically meaningful. 
Past studies focusing on aggregate analysis of all single cells in the population reported condition-specific dynamical behavior of Msn2, such as prolonged nuclear pulsing after glucose starvation versus a burst of activation before acclimating to osmotic stress (Hao and O'Shea 2012; Petrenko et al. 2013; AkhavanAghdam et al. 2016). Elegant studies by Hansen et al. used artificial activation of Msn2 (through chemical inhibition of PKA activity) to show that these differences in Msn2 nuclear translocation dynamics produce different transcriptional outputs (Hansen and O'Shea 2013; Hansen and O'Shea 2015a; Hansen and O'Shea 2015b; Hansen and O'Shea 2016). Target-gene promoters display different dependencies on the amplitude, frequency, and duration of Msn2 nuclear translocation, such that distinctions in Msn2 behavior activate different sets of genes. Comparable studies of regulators in mammalian systems also reported stress-specific differences in the dynamics of nuclear translocation, which correspond to differences in gene activation (Purvis et al. 2012; Kracikova et al. 2013; Paek et al. 2016). One limitation of the approach of Hansen et al. is that activating Msn2 by wholesale inhibition of PKA likely loses much of the heterogeneity seen in natural responses. Our study thus provides an important complement to artificial system activation.

In fact, our analysis revealed highly varied responses across subpopulations of cells responding to the same stress in the same environmental system: some cells responded to the osmotic/ionic stress induced by $\mathrm{NaCl}$ with a large nuclear pulse of Msn2 followed by near complete acclimation, as previously reported for sorbitol-induced osmotic stress - but other cells showed extensive and prolonged Msn2 fluctuations 
during the acclimation phase, akin to what has been reported for glucose starvation. These subpopulations are obscured by aggregate analysis but have important implications, since the different dynamics of Msn2 (and likely also Dot6) activation produce different transcriptomic outputs, even for cells responding to the same stressor in the same environment. This hypothesis is consistent with past work from our lab investigating single-cell transcriptomics, in which isogenic cells in the same culture displayed different transcriptomes upon $\mathrm{NaCl}$ stress, including for ESR genes, indicating that they experience the stress differently (Gasch et al. 2017).

An important unanswered question in biology is why some cells persist and later resume growing after what should be a lethal drug treatment, whereas other cells succumb. This has major medical importance, from bacterial cells that evade antibiotic treatment to cancer cells surviving chemotherapy to later relapse to growth and metastasis. Here, we found that a multi-factorial model of cell phenotypes could explain $35 \%$ of the variance in post-stress growth rate. Although Msn2 response had been previously linked to growth rate in single cells (Li et al. 2018), we found little predictive association with Msn2 behavior (Figure 6A).

Instead, the response of Dot6 explained a much larger fraction of the variance in post-stress growth rate, when considered alone or in the multi-factor linear model (Figure 6 and Table 3). Cells with a larger Dot6 response during the acute-stress phase had a faster recovery during the acclimation phase (Figure S2F). Dot6 is a repressor of ribosome-biogenesis genes, and this connection is intriguing since ribosome abundance is associated with faster growth rates in many (although not all) circumstances (Dai et al. 2016; Metzl-Raz et al. 2017; Dai et al. 2018; Ho et al. 2018). Whereas a cell's post- 
stress recovery rate is partly explained by its pre-stress growth rate (with $20 \%$ of the variance explained), its connection to the Dot6 acute-stress response is separable, since the two factors together explained a significantly higher fraction of the variance in recovery growth rate $(26 \%)$ than pre-stress growth rate did alone (See Model 5 in Table S3). We propose that the relationship between Dot6 response and post-stress growth rate lies in the production of ribosomes at the time of $\mathrm{NaCl}$, which can be separated from the growth rate at that moment. Cells with higher active transcription of ribosome biogenesis genes at the time of $\mathrm{NaCl}$ exposure may require stronger slamming-on of the transcriptional brakes, reflected in a larger Dot6 acute-stress response. In turn, cells with new ribosomes coming off the production line may be prepared for faster recovery after $\mathrm{NaCl}$ stress, even if their pre-stress growth rate was not already accelerated. This is known for bacterial cells responding to antibiotic stress, where cells with higher ribosome content at the time of stress relief resume faster, including cells not growing when exposed to stress/relief (Kim et al. 2018; Remigi et al. 2019).

Another major discovery of this work is that the regulation of Msn2 and Dot6, which together activate and repress gene sets coordinately induced and repressed in the yeast ESR, show less coordination than we expected at the outset. This suggests significant, unrealized differences in how the two factors are regulated during a stress response. Although the pre-stress activation patterns of Msn2 and Dot6 were correlated above random chance, many of the nuclear pulses were uncoupled in timing and magnitude. Furthermore, the acclimation profiles were quite different and often unrelated. These differences could emerge through several possibilities, including differences in attenuation/feedback and differences in upstream activation inputs. On 
one hand, levels of Dot6 nuclear localization completely acclimate to pre-stress levels in most cells. Previous theory demonstrated that acclimation can be achieved by only a few basic regulatory circuits (Ma et al. 2009), suggesting that Dot6 is regulated by a simple circuit whereas Msn2 acclimation may be controlled by more extensive feedback. On the other hand, the two factors could differ substantially in upstream regulation. Many signaling pathways have been implicated in Msn2 regulation, even during the same response (Mayordomo et al. 2002; Lee et al. 2013; Chasman et al. 2014; Gutin et al. 2015; Gutin et al. 2019; Mace et al. 2020); whether these pathways also regulate Dot6 is not known. In contrast, we found that Dot6 nuclear translocation before $\mathrm{NaCl}$ addition was significantly correlated in cells in the same colony, but Msn2 pulses were not, indicating that Dot6 may be responding to signals distinct from those activating Msn2. Future experiments interrogating Msn2 and Dot6 behavior in regulatory mutants will shed light on these possibilities.

The distinct responses of Msn2 and Dot6 may be informative to cells: the relative pre-stress activation of Msn2 versus Dot6 improved the fit with Msn2 acutestress response (and in one strain, with the Dot6 acute-stress response), and in both strains was even more predictive of the relative activation of Msn2 and Dot6 during stress. The relative pre-stress activation of Msn2 versus Dot6 did not improve correlations with growth rate or other cellular features, arguing against a technical explanation. We propose that cell-to-cell differences in pre-stress Msn2 and Dot6 behavior may reflect subtle distinctions in cellular states, and these distinctions influence the subsequent stress response. We show that these states are unlikely to relate strongly to cell-cycle phase or cell size, but could reflect different metabolic states 
known from single-cell microscopy and transcriptomic studies (Gasch et al. 2017;

Bagamery et al. 2020). While the exact nature is not presently known, together these results highlight that much of the heterogeneity in stress responses is predictable and determined by a cell's prior life history.

\section{Methods}

Strain AGY1328 (BY1471 DOT6-GFP(S65T)-His3MX, MSN2-mCherry-HYGMX) was used for most analyses. Trends in transcription factor behavior were validated with strain AGY1666 (BY1471 MSN2-GFP(S65T)-His3MX, DOT6-mCherry-HYGMX). For microscopy experiments, overnight cultures were grown from single colonies to exponential phase (Optical Density, $\mathrm{OD}_{600}<1$ ) in Low Fluorescent Medium (LFM) before cells were adhered to the microscope slide as described below. LFM consisted of $0.17 \%$ Yeast Nitrogen Base without Ammonium Sulfate, Folic Acid, or Riboflavin (\#MP114030512, Thermo Fisher Scientific, Waltham, Massachusetts), 0.5\% Ammonium Sulfate, $0.2 \%$ complete amino acids supplement, where individual amino acids concentrations are as defined in Yeast Synthetic Drop-out Media Supplements (SigmaAldrich, Saint Louis, Missouri), and 2\% Glucose.

An FCS2 chamber (Bioptechs Inc, Butler, Pennsylvania) microfluidic system was used for time-lapse microscopy. In short, a $40 \mathrm{~mm}$ round glass coverslip and FCS2 lower gasket were assembled, and Concanavalin A solution ( $2 \mathrm{mg} / \mathrm{mL}$ Concanavalin $\mathrm{A}$, $5 \mathrm{mM} \mathrm{MnCl}_{2}, 5 \mathrm{mM} \mathrm{CaCl}_{2}$ ) was applied to the coverslip, incubated for two minutes, then aspirated. Next, $350 \mu \mathrm{L}$ of an $\sim 0.5 \mathrm{OD}_{600}$ culture was placed on the coverslip and 
incubated 5 minutes for cells to settle and adhere to the Concanavalin A. $150 \mu \mathrm{L}$ of the media was then removed and the rest of the FCS2 chamber was assembled.

Media was flown through the FCS2 chamber using gravity flow. Input tubing was attached to elevated bottles containing either LFM or LFM $+0.7 \mathrm{M} \mathrm{NaCl}$ (See diagram in Figure 1B) with a valve to switch between media with and without $0.7 \mathrm{M} \mathrm{NaCl}$. The outflow tubing was connected to an additional $\sim 1$ meter of BD Intramedic PE Tubing (\#1417012D, Thermo Fisher Scientific, Waltham, Massachusetts) with the smaller inner diameter of $0.86 \mathrm{~mm}$ being vital to controlling the gravity flow of media. The entire assembly, including the microscope stand, bottles containing media, and FCS2 chamber, were enclosed in an incubator maintaining internal temperature of $30^{\circ} \mathrm{C}$ throughout the entire protocol.

A Nikon Eclipse Ti inverted microscope with the Perfect Focus System (Nikon Inc., Melville, New York ) was used for time-lapse microscopy. The GFP signal was captured using a ET-EGFP single band filter cube (\#49002, Chroma Technology Corp, Bellows Falls, Vermont) with an excitation filter centered on 470 and a $40 \mathrm{~nm}$ bandwidth. The mCherry signal was captured using a $\mathrm{ET} / \mathrm{mCH} / \mathrm{TR}$ single band filter cube (\#96365, Chroma Technology Corp, Bellows Falls, Vermont) with an excitation filter centered on $560 \mathrm{~nm}$ with a $40 \mathrm{~nm}$ bandwidth. In addition, exposure from a halogen lamp was used to capture white-light images of all cells.

Images of each field of view were captured at 6-minute intervals. The z-focal plane focus was set on the center of cells, and images were taken 1 um above, at, and 1 um below this center of focus, generating a three-image z-stack for each channel. The 
three-image z-stacks were collapsed into a single image by taking the maximum projection of the 3 images using a custom MATLAB script.

Cells were identifying using a MATLAB circle-finding function on the brightfield images. Individual cells were then tracked through all images using the MATLAB simpletracker function (Tinevez 2019). Cell colonies were defined by segmenting images into a binary black-and-white image, and single colonies were defined as enclosed masks. The number of cells within each colony was determined simply as the number of identified circles that overlapped with a given enclosed white area of the binary images. Pre-stress growth was scored by linear regression on colony size (defined as the total pixel number within the masked area of the colony) for the first twelve 6-minute time points and reported as the natural log of the rate of increase. Poststress growth was measured in the same manner for time points 20-29 (representing resumed growth at the beginning of the acclimation phase: 114-168 minutes into the time course).

We applied several quantity control filters to insure accuracy of growth rates. First, to insure that colony growth rates were representative of nuclear localization dynamics within individual cells, we limited our analysis to colonies consisting of no more than two cells at the time points leading up to $\mathrm{NaCl}$ exposure. Most of these twocell colonies represented mother/daughter cells and therefore had clear shared life histories. Second, in some cases a budding daughter cell was lost during the timecourse, resulting in a misleading negative growth rate. Consequently, regressions resulting in negative slopes were excluded. Lastly, a visual inspection of individual colonies during the time course excluded colonies where new cells adhered to a given 
colony. Thirty cells were excluded from post-stress measures due to these cell adhesion issues that skewed colony size measures. Another six cells ( $2.7 \%$ of total cells) had no apparent post-stress growth and the calculated slope was therefore dominated by noise in pixel number. This resulted in either a negative or near zero slope and consequently did not provide an informative growth rate measure when taking the natural log of the change in colony size. Consequently, these six cells were also excluded from poststress growth rate measures.

Cell-cycle phase at the time of osmotic stress was measured by visual inspection of cell bud presence/size and nucleus location within the cell in accordance with standard yeast cell-cycle definitions (Howell and Lew 2012). Specifically, S-phase) appearance of a bud but no migration of nucleus, G2) bud and nucleus migration toward bud, but no nucleus in daughter cell, M-phase) nucleus in both cell and bud, and active division of nuclei, G1) no bud and nucleus is not actively dividing.

Nuclear localization of Msn2 and Dot6 was measured by taking the pixel intensity of the top $5 \%$ of pixels in the cell divided by the median pixel intensity within the circle mask identified for each cell, similar to other studies (Cai et al. 2008; Hao and O'Shea 2012; Petrenko et al. 2013; Lin et al. 2015; AkhavanAghdam et al. 2016; Gasch et al. 2017; Granados et al. 2018). The following nuclear localization metrics were analyzed:

* Nuclear localization Peaks: Temporal peaks of nuclear localization were identified using the MATLAB findpeaks function, where a peak height is called from a local maximum to the nearest minimum ('valley') on either side of the peak. In order to estimate a threshold for a true peak of nuclear localization versus background noise, a 
linear regression was done on pre-stress nuclear localization time points to calculate the difference of each point from the regression line, resulting in a baseline standard deviation of localization values. Since from visual inspection of traces and cells there were many more true peaks for Dot6, the standard deviation for the Msn2-mCherry channel was used to calculate this baseline threshold for both Msn2 and Dot6. Specifically, two standard deviations from the mean of the distribution of was used as a threshold. This threshold appeared to be accurate by visual inspection of cells, where the threshold distinguished what looked like true nuclear localization from the images.

* Area Under the Curve (AUC) of nuclear localization: For pre-stress time points, AUC was calculated by summing the first 9 measurements of nuclear localization scores (top brightest $5 \%$ of pixels over the median cellular signal). This summation represents the total relative levels of nuclear localization between all cells. The same AUC calculation was done for the acclimation phase using time points $24-37$. The difference in AUC between the two signals (Msn2 - Dot6 AUC in Figures 6 and 7) is simply the difference of the two individual AUC measurements.

* Acute Stress Peak Height: The acute stress peak height was calculated by taking the maximum of nuclear localization score during the acute stress response (time points $13-20$ ) and then subtracting the minimum of the nuclear localization scores just before stress (time points $11-13$ ).

\section{Cell clustering to identify subpopulations}

Nuclear localization scores were $\log _{2}$ transformed, and for each cell and each factor, the value at each timepoint was normalized to the median of all cells for that factor and time 
point (Figure 4, blue/yellow scale data). The population-median-normalized vector for Msn2 and Dot6 were concatenated and clustered by mclust (Scrucca et al. 2016) using model Ell and $k=30$ (which was collapsed to $k=11$ by mclust). The $\log _{2}$ of unnormalized nuclear traces for each cell was added for display in Figure 4 (orange/white scale data). Relationships with logged growth rate data before and after stress, calculated as described above, were scored for each cluster of cells compared to all other cells in the data (Figure 5, Wilcoxon Rank Sum test).

Visual inspection of cells within mclust clusters 2 and 3 indicated that some Msn2 signal was focused but outside the nucleus (evidence after $\mathrm{NaCl}$ treatment), likely in the vacuole. There were 18 cells were this was observed visually. Since the impact of this signal was uncertain, these cells were excluded from subsequent regression modeling (i.e. Figures 5A, 6 and 7).

Probabilities of the number cells from each of the three biological replicates.

Binomial probabilities were used to determine if each cluster contained more cells from one of the three biological replicates than would be expected by chance. Specifically, if $x$ is the number of cells from a given biological replicate present in a cluster, then probability of having $x$ cells or more in the cluster is

$$
\operatorname{Pr}(\mathrm{X} \geq \mathrm{x})=\sum_{k=X}^{n}\left(\begin{array}{l}
n \\
k
\end{array}\right) p^{k}(1-p)^{n-k}
$$


where $n$ is the number of cells in the cluster and $p$ is the expected probability of having a cell from a given replicates (that is, the total number of cells in the replicate divided by the total number of cells in all three replicates). Since clusters 8 and 10 had a total of 5 and 6 cells, respectively, they lacked statistical power and were excluded from the analysis.

The Holm-Bonferroni method was used for multiple hypotheses correction, where there were $n=27$ ( 9 clusters and testing the number of cells from 3 biological replicates in each cluster) and $\alpha=0.05$. Using this threshold, only Cluster 9 showed strong enrichment for cells from one replicate compared to expected after multiple-test correction. Of note, this was the only cluster (besides Clusters 8 and 10), that had zero cells from a given replicate (Table S1).

\section{Permutations of nuclear localization peak matches}

To identify if matched peaks of Msn2 and Dot6 were more coordinated than expected by chance, permutations were performed where a random Msn2 and Dot6 trace, including the time points of the called peaks, were randomly paired from the entire dataset. Coordinated peaks were then calculated from these random Msn2/Dot6 trace pairs. These permutations indicated that the number of matched Msn2/Dot6 peaks per cell was much higher than expected by random combinations (zero permuted datasets out of 100,000 total had 0.21 matched pre-stress peaks per cell or more). The same test was done for the matched peaks during the acclimation phase, and although the number of matched peaks per cell was significant for the acclimation time points (a fraction of $4 \times 10^{-4}$ of permuted datasets had 0.07 matched peaks per cell or more), this was significantly less than that for the pre-stress time points. This again demonstrated 
that there was more coordination in nuclear localization between Msn2 and Dot6 during the before stress compared to after stress.

Permutations of nuclear localization peak correlations between cells in two-cell colonies

There were 56 two-cell colonies in the dataset. Of these, 15 colonies showed coordinated Dot6 peaks between the two cells, defined as peaks occurring within one time point of each other. Permutations were performed where the 112 cells from these 56 colonies were randomly assigned in pairs and the same coordinated peak measurements were performed. Similarly, permutations were performed on matched peaks of Msn2. Results are shown in Table S3.

\section{Linear models}

Multiple linear regressions shown in Figure 6 and Table S3 were performed using fit/m in MATLAB. For each model was represented by

$$
y=\beta_{0}+\beta_{1} x_{1}+\beta_{2} x_{2}+\cdots+\beta_{n} x_{n}+\varepsilon
$$

where the dependent variable $y$ is the post-stress growth rate, $\beta_{0}$ is the intercept, each subsequent $\beta$ is the estimate of the slope for each independent variable $x$, and $\varepsilon$ is the error term. A list of independent variables is shown in Table S3 for each of the multiple linear regression performed. The p-values shown in Table S3 were determined from the t-statistic of each $\beta$ coefficient was not equal to zero. In Table S3, Model 1 included all variables in the model. Model 2 only included the significant independent variables from Model 1. Model 3 excluded Msn2 acclimation AUC and Cell/colony size from Model 2 as the $\mathrm{p}$-values did not pass Holm-Bonferroni correction ( $\alpha=0.05$ and $n=14)$. Since 
Model 3 gave the four most-significant variables, Model 4 then removed pre-stress growth rate to see the resulting explained variance. Model 5 measured the explained variance of the two most significant variables: Dot6 acute stress peak height and prestress growth rate.

\section{References:}

AkhavanAghdam Z, Sinha J, Tabbaa OP, Hao N. 2016. Dynamic control of gene regulatory logic by seemingly redundant transcription factors. Elife 5 .

Bagamery LE, Justman QA, Garner EC, Murray AW. 2020. A Putative Bet-Hedging

Strategy Buffers Budding Yeast against Environmental Instability. Curr. Biol. 30.

Balaban NQ, Merrin J, Chait R, Kowalik L, Leibler S. 2004. Bacterial persistence as a phenotypic switch. Science (80-. ). 305.

Bergenholm D, Liu G, Holland P, Nielsen J. 2018. Reconstruction of a Global

Transcriptional Regulatory Network for Control of Lipid Metabolism in Yeast by Using Chromatin Immunoprecipitation with Lambda Exonuclease Digestion. mSystems 3.

Brauer MJ, Huttenhower C, Airoldi EM, Rosenstein R, Matese JC, Gresham D, Boer VM, Troyanskaya OG, Botstein D. 2008. Coordination of growth rate, cell cycle, stress response, and metabolic activity in yeast. Mol. Biol. Cell 19.

Cai L, Dalal CK, Elowitz MB. 2008. Frequency-modulated nuclear localization bursts 
coordinate gene regulation. Nature 455.

Castrillo JI, Zeef LA, Hoyle DC, Zhang N, Hayes A, Gardner DCJ, Cornell MJ, Petty J,

Hakes L, Wardleworth L, et al. 2007. Growth control of the eukaryote cell: A

systems biology study in yeast. J. Biol. 6 .

Causton HC, Ren B, Koh SS, Harbison CT, Kanin E, Jennings EG, Lee TI, True HL,

Lander ES, Young RA. 2001. Remodeling of Yeast Genome Expression in

Response to Environmental Changes. Mol. Biol. Cell.

Chasman D, Ho YH, Berry DB, Nemec CM, MacGilvray ME, Hose J, Merrill AE, Lee M

V, Will JL, Coon JJ, et al. 2014. Pathway connectivity and signaling coordination in

the yeast stress-activated signaling network. Mol Syst Biol.

Cheong R, Rhee A, Wang CJ, Nemenman I, Levchenko A. 2011. Information

transduction capacity of noisy biochemical signaling networks. Science (80-. ). 334 .

Crane MM, Clark IBN, Bakker E, Smith S, Swain PS. 2014. A microfluidic system for

studying ageing and dynamic single-cell responses in budding yeast. PLoS One 9.

Dai X, Zhu M, Warren M, Balakrishnan R, Okano H, Williamson JR, Fredrick K, Hwa T.

2018. Slowdown of translational elongation in Escherichia coli under hyperosmotic stress. MBio 9.

Dai X, Zhu M, Warren M, Balakrishnan R, Patsalo V, Okano H, Williamson JR, Fredrick K, Wang YP, Hwa T. 2016. Reduction of translating ribosomes enables Escherichia coli to maintain elongation rates during slow growth. Nat. Microbiol. 2.

Fehrmann S, Paoletti C, Goulev Y, Ungureanu A, Aguilaniu H, Charvin G. 2013. Aging yeast cells undergo a sharp entry into senescence unrelated to the loss of mitochondrial membrane potential. Cell Rep. 5. 
Gasch AP, Spellman PT, Kao CM, Carmel-Harel O, Eisen MB, Storz G, Botstein D,

Brown PO. 2000. Genomic Expression Programs in the Response of Yeast Cells to Environmental Changes. Mol. Biol. Cell.

Gasch AP, Yu FB, Hose J, Escalante LE, Place M, Bacher R, Kanbar J, Ciobanu D, Sandor L, Grigoriev I V., et al. 2017. Single-cell RNA sequencing reveals intrinsic and extrinsic regulatory heterogeneity in yeast responding to stress. PLOS Biol. Görner W, Durchschlag E, Martinez-Pastor MT, Estruch F, Ammerer G, Hamilton B, Ruis H, Schüller C. 1998. Nuclear localization of the $\mathrm{C} 2 \mathrm{H} 2 z$ inc finger protein Msn2p is regulated by stress and protein kinase A activity. Genes Dev.

Granados AA, Pietsch JMJ, Cepeda-Humerez SA, Farquhar IL, Tkačik G, Swain PS.

2018. Distributed and dynamic intracellular organization of extracellular information.

Proc. Natl. Acad. Sci.

Gutin J, Joseph-Strauss D, Sadeh A, Shalom E, Friedman N. 2019. Genetic screen of the yeast environmental stress response dynamics uncovers distinct regulatory phases. Mol. Syst. Biol. 15.

Gutin J, Sadeh A, Rahat A, Aharoni A, Friedman N. 2015. Condition-specific genetic interaction maps reveal crosstalk between the cAMP / PKA and the HOG MAPK pathways in the activation of the general stress response . Mol. Syst. Biol. 11.

Hansen AS, O'Shea EK. 2013. Promoter decoding of transcription factor dynamics involves a trade-off between noise and control of gene expression. Mol. Syst. Biol. Hansen AS, O'Shea EK. 2015a. Cis Determinants of Promoter Threshold and Activation Timescale. Cell Rep.

Hansen AS, O'Shea EK. 2015b. Limits on information transduction through amplitude 
and frequency regulation of transcription factor activity. Elife.

Hansen AS, O'Shea EK. 2016. Encoding four gene expression programs in the activation dynamics of a single transcription factor. Curr. Biol.

Hansen AS, Zechner C. 2021. Promoters adopt distinct dynamic manifestations depending on transcription factor context. Mol. Syst. Biol. 17.

Hao N, O'Shea EK. 2012. Signal-dependent dynamics of transcription factor translocation controls gene expression. Nat. Struct. Mol. Biol.

Ho YH, Shishkova E, Hose J, Coon JJ, Gasch AP. 2018. Decoupling Yeast Cell Division and Stress Defense Implicates mRNA Repression in Translational Reallocation during Stress. Curr. Biol. 28.

Howell AS, Lew DJ. 2012. Morphogenesis and the cell cycle. Genetics 190. Inde Z, Dixon SJ. 2018. The impact of non-genetic heterogeneity on cancer cell death. Crit. Rev. Biochem. Mol. Biol. 53.

Jin M, Li Y, O'Laughlin R, Bittihn P, Pillus L, Tsimring LS, Hasty J, Hao N. 2019. Divergent Aging of Isogenic Yeast Cells Revealed through Single-Cell Phenotypic Dynamics. Cell Syst. 8.

Keren I, Shah D, Spoering A, Kaldalu N, Lewis K. 2004. Specialized persister cells and the mechanism of multidrug tolerance in Escherichia coli. J. Bacteriol. 186.

Kim JS, Yamasaki R, Song S, Zhang W, Wood TK. 2018. Single cell observations show persister cells wake based on ribosome content. Environ. Microbiol. 20.

Kracikova M, Akiri G, George A, Sachidanandam R, Aaronson SA. 2013. A threshold mechanism mediates p53 cell fate decision between growth arrest and apoptosis. Cell Death Differ. 20. 
Lee MCW, Lopez-Diaz FJ, Khan SY, Tariq MA, Dayn Y, Vaske CJ, Radenbaugh AJ, Kim HJ, Emerson BM, Pourm N. 2014. Single-cell analyses of transcriptional heterogeneity during drug tolerance transition in cancer cells by RNA sequencing. Proc. Natl. Acad. Sci. U. S. A. 111.

Lee P, Kim MS, Paik SM, Choi SH, Cho BR, Hahn JS. 2013. Rim15-dependent activation of Hsf1 and Msn2/4 transcription factors by direct phosphorylation in Saccharomyces cerevisiae. FEBS Lett. 587.

Levy SF, Ziv N, Siegal ML. 2012. Bet hedging in yeast by heterogeneous, agecorrelated expression of a stress protectant. PLOS Biol.

Li S, Giardina DM, Siegal ML. 2018. Control of nongenetic heterogeneity in growth rate and stress tolerance of Saccharomyces cerevisiae by cyclic AMP-regulated transcription factors. PLoS Genet. 14.

Lin Y, Sohn CH, Dalal CK, Cai L, Elowitz MB. 2015. Combinatorial gene regulation by modulation of relative pulse timing. Nature 527:54-58.

Lippman SI, Broach JR. 2009. Protein kinase A and TORC1 activate genes for ribosomal biogenesis by inactivating repressors encoded by Dot6 and its homolog Tod6. Proc. Natl. Acad. Sci. U. S. A.

Lu C, Brauer MJ, Botstein D. 2009. Slow Growth Induces Heat-Shock Resistance in Normal and Respiratory-deficient Yeast. Mol. Biol. Cell.

Ma W, Trusina A, El-Samad H, Lim WA, Tang C. 2009. Defining Network Topologies that Can Achieve Biochemical Adaptation. Cell 138.

Mace K, Krakowiak J, El-Samad H, Pincus D. 2020. Multi-kinase control of environmental stress responsive transcription. PLoS One 15. 
Mayordomo I, Estruch F, Sanz P. 2002. Convergence of the target of rapamycin and the Snf1 protein kinase pathways in the regulation of the subcellular localization of Msn2, a transcriptional activator of STRE (stress response element)-regulated genes. J. Biol. Chem. 277.

Metzl-Raz E, Kafri M, Yaakov G, Soifer I, Gurvich Y, Barkai N. 2017. Principles of cellular resource allocation revealed by condition-dependent proteome profiling. Elife 6.

Paek AL, Liu JC, Loewer A, Forrester WC, Lahav G. 2016. Cell-to-Cell Variation in p53 Dynamics Leads to Fractional Killing. Cell 165.

Petrenko N, Chereji R V., McClean MN, Morozov A V., Broach JR. 2013. Noise and interlocking signaling pathways promote distinct transcription factor dynamics in response to different stresses. Mol. Biol. Cell.

Purvis JE, Karhohs KW, Mock C, Batchelor E, Loewer A, Lahav G. 2012. p53 dynamics control cell fate. Science (80-. ).

Purvis JE, Lahav G. 2013. Encoding and decoding cellular information through signaling dynamics. Cell 152.

Regenberg B, Grotkjær T, Winther O, Fausbøll A, Åkesson M, Bro C, Hansen LK, Brunak S, Nielsen J. 2006. Growth-rate regulated genes have profound impact on interpretation of transcriptome profiling in Saccharomyces cerevisiae. Genome Biol. 7.

Remigi P, Ferguson GC, McConnell E, De Monte S, Rogers DW, Rainey PB. 2019. Ribosome Provisioning Activates a Bistable Switch Coupled to Fast Exit from Stationary Phase. Mol. Biol. Evol. 36. 
Scrucca L, Fop M, Murphy TB, Raftery AE. 2016. Mclust 5: Clustering, classification and density estimation using Gaussian finite mixture models. $R$ J. 8.

Shaffer SM, Dunagin MC, Torborg SR, Torre EA, Emert B, Krepler C, Beqiri M, Sproesser K, Brafford PA, Xiao M, et al. 2017. Rare cell variability and druginduced reprogramming as a mode of cancer drug resistance. Nature 546.

Smith A, Ward MP, Garrett S. 1998. Yeast PKA represses Msn2p/Msn4p-dependent gene expression to regulate growth, stress response and glycogen accumulation. $E M B O \mathrm{~J}$.

Stewart-Ornstein J, Nelson C, Derisi J, Weissman JS, El-Samad H. 2013. Msn2 coordinates a stoichiometric gene expression program. Curr. Biol.

Tinevez J-Y. 2019. simpletracker. Available from:

https://www.mathworks.com/matlabcentral/fileexchange/34040-simpletracker Zakrzewska A, Van Eikenhorst G, Burggraaff JEC, Vis DJ, Hoefsloot H, Delneri D, Oliver SG, Brul S, Smits GJ. 2011. Genome-wide analysis of yeast stress survival and tolerance acquisition to analyze the central trade-off between growth rate and cellular robustness. Mol. Biol. Cell 22.

Zaman S, Lippman SI, Schneper L, Slonim N, Broach JR. 2009. Glucose regulates transcription in yeast through a network of signaling pathways. Mol. Syst. Biol. Zaman S, Lippman SI, Zhao X, Broach JR. 2008. How Saccharomyces Responds to Nutrients. Annu. Rev. Genet. 
Supplemental Figures and Tables
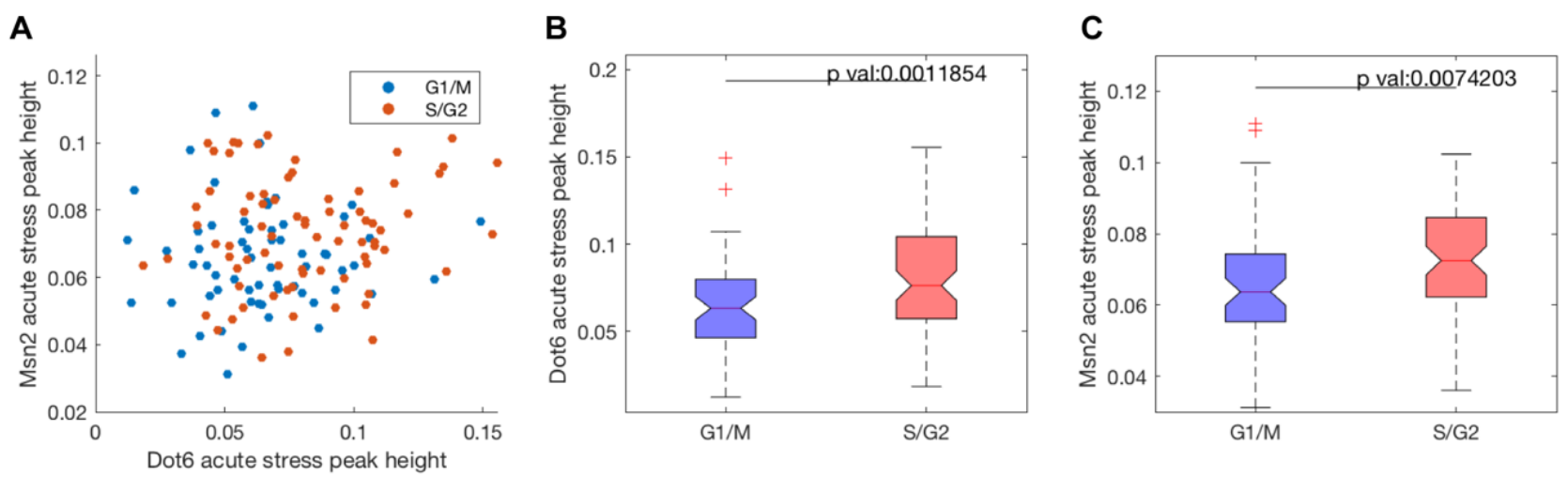

Figure S1. The strength of transcription factor nuclear localization is weakly related to cell cycle phase and budding. Cells grow the fastest in G1 and M-phase (Goranov et al., 2009), thus cells were binned if they were in G1 or M phase or S/G2 phases at the time of $\mathrm{NaCl}$ exposure. A. The peak height of nuclear localization for Msn2 and Dot6 during the acute-stress phase are plotted, with cells in S/G2 or M/G1 indicated in red or blue, respectively. B. and C. The distribution of acute-stress peak heights for Dot6 (B) or Msn2 (C) was plotted for cells in M/G1 or S/G2. Wilcoxon rank sum tests show that cells in S/G2 had slightly higher nuclear accumulation of both factors $(p<0.001)$. 

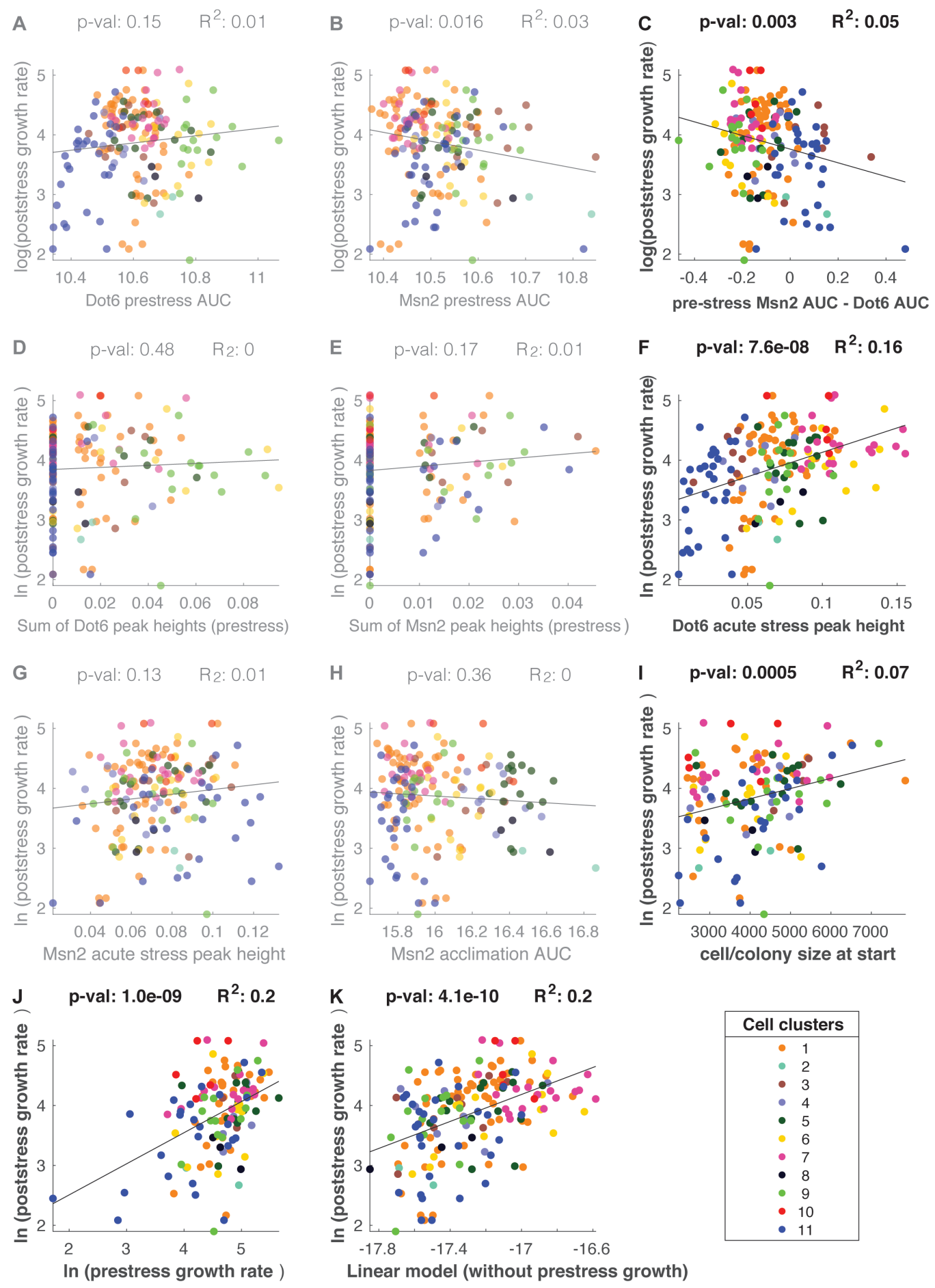

Figure S2. Linear regression of individual parameters on post-stress growth rate. (A-J). Linear regressions of individual parameters listed in Table S2. Parameters in which the false discovery rate was $<0.025(p<0.003)$ are bold whereas other plots are deemphasized. There is a significant fit between cell/colony size at the experiment start time and post-stress growth rate (I); however, this parameter was not significant beyond the multiple-test threshold in the multi-factor linear model (see Table 2), 
suggesting that much of cell-size contribution is correlated with and thus absorbed by other factors in the model. K. Fit from a multiple linear model similar to that shown in Figure 6 except in which pre-stress growth rate was not included (coefficient set to 0 ). The figure demonstrates that a substantial amount of the variation in post-stress growth rate is explained by features other than pre-stress growth rate of the cells.

A

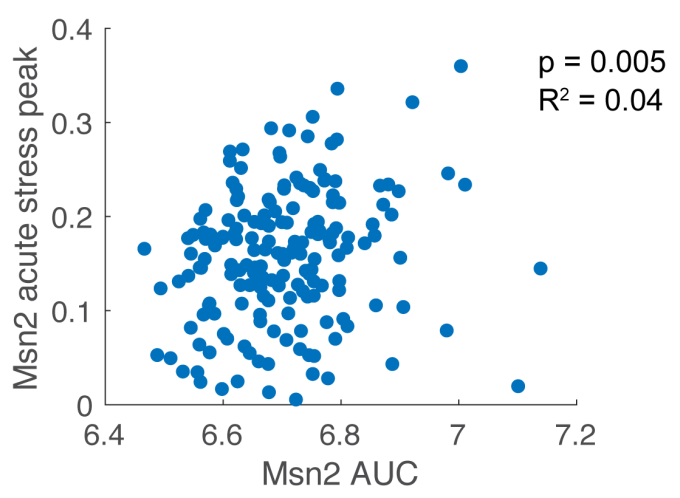

C

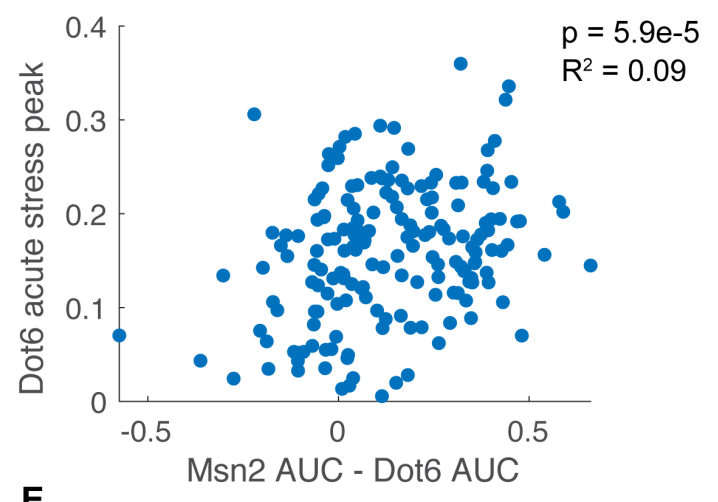

E

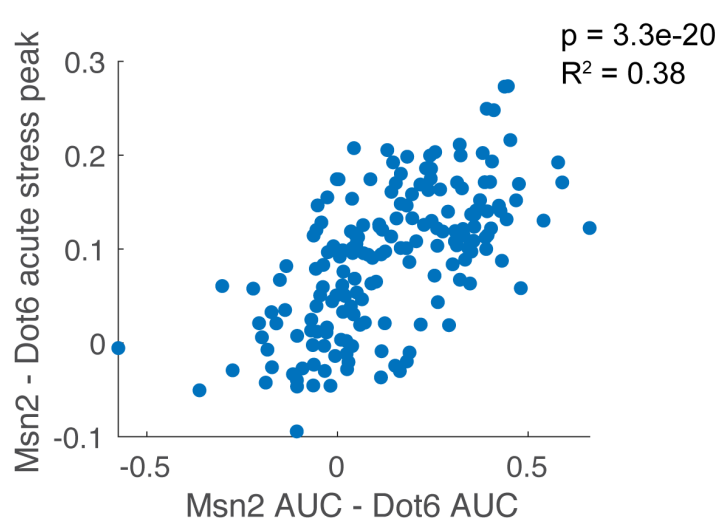

B

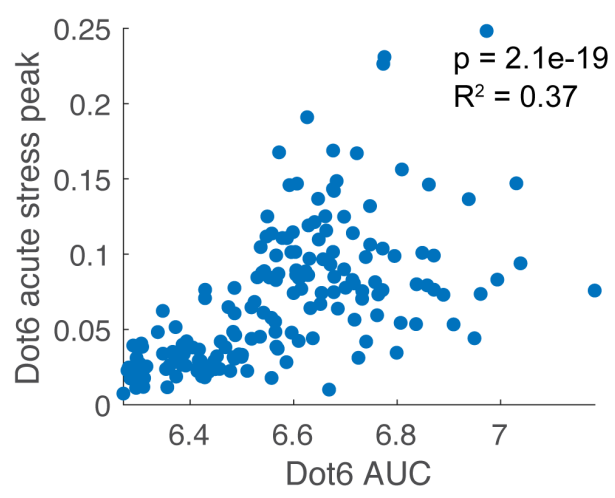

D

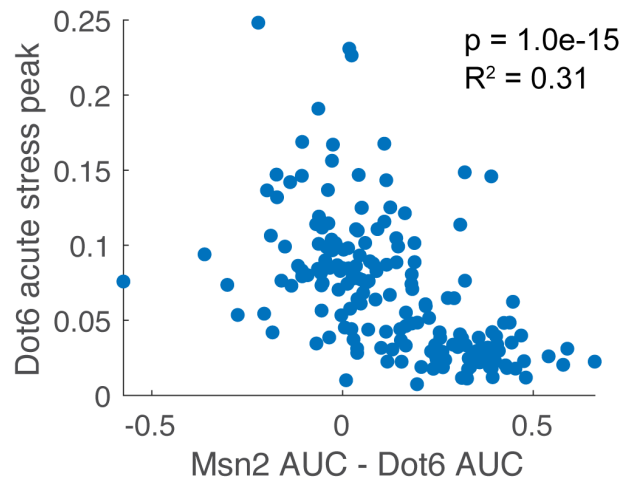

Figure S3. Comparison of pre-stress Area Under the Curve with acute stress peak height. Same as Figure 7 in main text, but for strain AGY1666 (Msn2-GFP, Dot6mCherry). Acute stress peak heights and pre-stress area under the curve (AUC) are as defined in Figure 6B in main text. Here, two experimental batches done with strain AGY1666 were combined. Here, (D) shows that taking the difference between Msn2 
and Dot6 pre-stress AUC does not improve the correlation as it had done for strain AGY (Figure 7D in main text). However, there is a slight improvement when the pre-stress AUC difference is correlated with the difference in acute stress peak heights $(E)$.

A

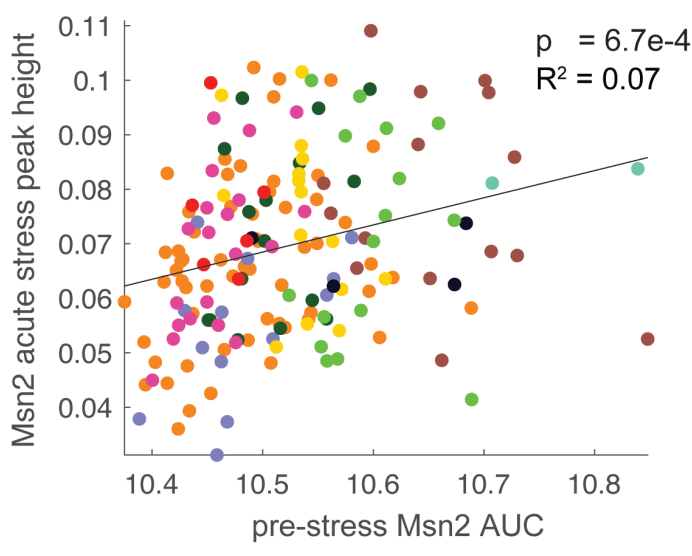

C

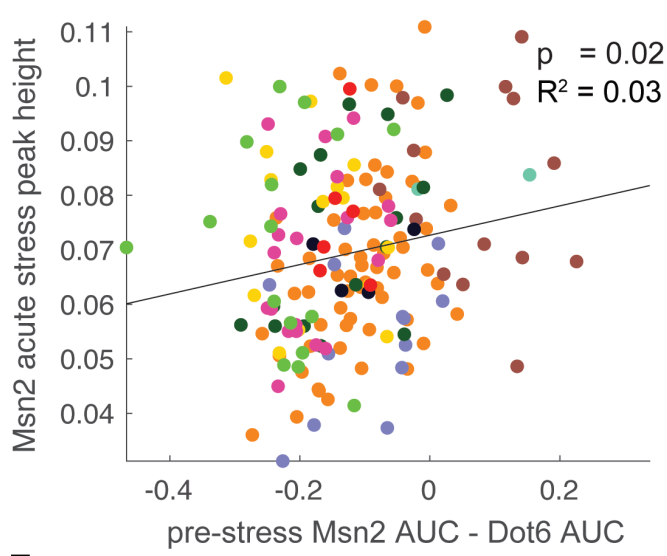

E

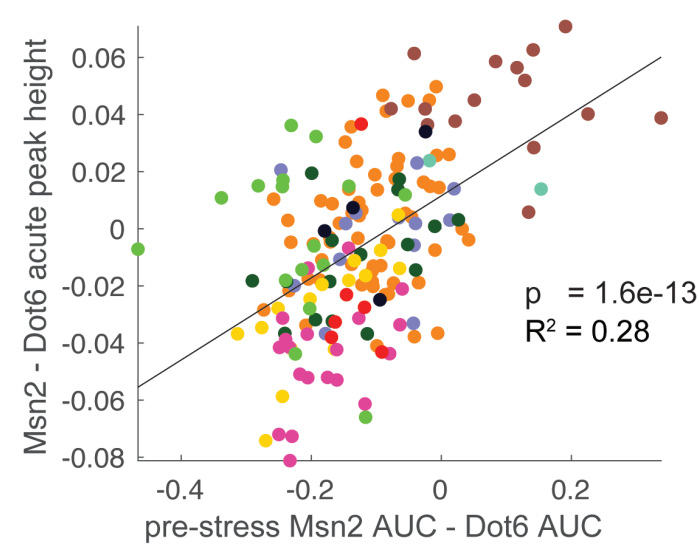

B
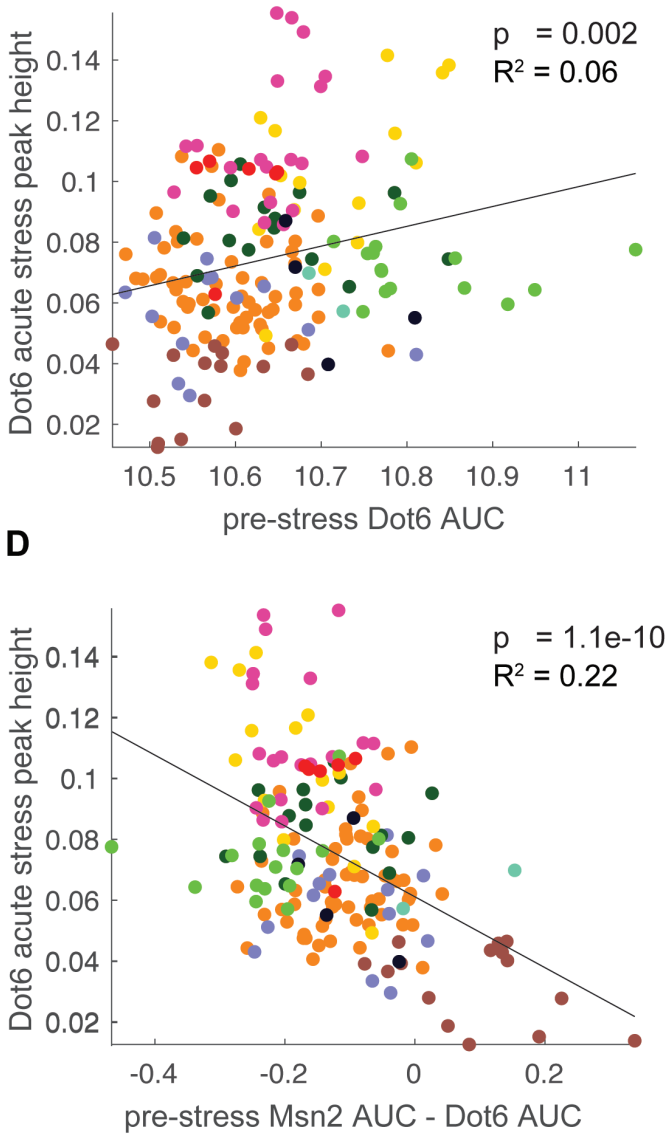

\begin{tabular}{|c|}
\hline Cell clusters \\
\hline$\bullet 1$ \\
$\bullet 2$ \\
$\bullet 3$ \\
$\bullet 4$ \\
$\bullet 5$ \\
$\bullet 6$ \\
$\bullet 7$ \\
$\bullet 8$ \\
$\bullet$ \\
$\bullet 10$ \\
$\bullet 11$ \\
\hline
\end{tabular}

Figure S4. Cells in Cluster 11 do not explain correlations in transcription factor behavior. As shown in Figure 7 except with cells from cell Cluster 11 removed from the analysis. 
Table S1. Permutations of coordinately timed peaks in cells in two-cell colonies

\begin{tabular}{lll}
\cline { 2 - 3 } & $\begin{array}{c}\text { number of colonies with } \\
\text { coordinated peaks } \\
\text { (out of } 56 \text { total) }\end{array}$ & $\begin{array}{c}\text { Fraction of permutations with coordinated } \\
\text { peak number equal or greater to actual } \\
\text { number of colonies with coordinated } \\
\text { peaks }\end{array}$ \\
\hline $\begin{array}{l}\text { Dot6 coordinated } \\
\text { peak }\end{array}$ & 15 & $9.5 \times 10^{-4}$ \\
$\begin{array}{l}\text { Msn2 coordinated } \\
\text { peak }\end{array}$ & 6 & 0.19 \\
\hline
\end{tabular}

${ }^{*}$ There were 50,000 iterations of permutations for each comparison.

Table S2. Cell subpopulations are identified in multiple biological replicates

\begin{tabular}{llllllll} 
& & \multicolumn{3}{c}{$\begin{array}{c}\text { Cell number from each biological } \\
\text { replicate }\end{array}$} & \multicolumn{2}{c}{ P-value (before Holm-Bonferroni } \\
mclust & $\begin{array}{l}\text { Number } \\
\text { of cells in } \\
\text { cluster }\end{array}$ & $\begin{array}{l}\text { Biological } \\
\text { replicate 1 }\end{array}$ & $\begin{array}{l}\text { Biological } \\
\text { replicate 2 }\end{array}$ & $\begin{array}{l}\text { Biological } \\
\text { replicate 3 }\end{array}$ & $\begin{array}{l}\text { Biological } \\
\text { replicate 1 }\end{array}$ & $\begin{array}{l}\text { Biological } \\
\text { replicate 2 }\end{array}$ & $\begin{array}{l}\text { Biological } \\
\text { replicate 3 }\end{array}$ \\
\hline 1 & 61 & 23 & 21 & 17 & 0.46 & 0.13 & 0.94 \\
2 & 9 & 3 & 3 & 3 & 0.69 & 0.46 & 0.69 \\
3 & 18 & 7 & 9 & 2 & 0.50 & 0.034 & 1.00 \\
4 & 13 & 3 & 7 & 3 & 0.90 & 0.038 & 0.90 \\
5 & 22 & 10 & 4 & 8 & 0.25 & 0.89 & 0.58 \\
6 & 16 & 3 & 2 & 11 & 0.96 & 0.96 & 0.0086 \\
7 & 20 & 5 & 3 & 12 & 0.90 & 0.94 & 0.027 \\
8 & 5 & 1 & 0 & 4 & 0.90 & 1.00 & 0.062 \\
9 & 16 & 3 & 0 & 13 & 0.96 & 1.00 & $0.0003^{*}$ \\
10 & 6 & 4 & 1 & 1 & 0.13 & 0.85 & 0.93 \\
11 & 35 & 17 & 11 & 7 & 0.094 & 0.35 & 0.99 \\
\hline
\end{tabular}

${ }^{*}$ Significant after Holm-Bonferroni correction.

The number of cells in each mclust cluster from Figure 4 is shown along with the number of those cells from each of three biological replicates. P-values from binomial probability tests (see Methods) are shown and those significant after Holm-Bonferroni correction (namely Cluster 9 which was enriched for cells from replicate 3 ) are indicated with an asterisk. 
Table S3. Multiple Linear Models: variables, significance, and explained variance.

\begin{tabular}{|c|c|c|c|c|c|}
\hline \multirow[b]{2}{*}{ Model Parameter } & \multicolumn{5}{|c|}{$\begin{array}{c}\text { Multiple Linear Model } \\
\text { (p-values of included variables) }\end{array}$} \\
\hline & Model 1 & Model $2^{* *}$ & Model $3^{* * *}$ & Model 4 & Model 5 \\
\hline Intercept & $3.7 E-04$ & 8.9E-07 & $3.1 \mathrm{E}-05$ & 0.0009 & $1.75 \mathrm{E}-05$ \\
\hline Dot6 pre-stress AUC & 0.016 & 0.0004 & 0.00011 & 0.0070 & \\
\hline Msn2 pre-stress AUC & $\mathrm{NaN}$ & & & & \\
\hline Msn2 - Dot6 AUC & 0.468 & & & & \\
\hline $\begin{array}{l}\text { Sum of Dot6 peak heights } \\
\text { (pre-stress) }\end{array}$ & 0.767 & & & & \\
\hline $\begin{array}{l}\text { Sum of Msn2 peak heights } \\
\text { (pre-stress) }\end{array}$ & 0.016 & 0.0024 & 0.00010 & 0.0007 & \\
\hline Dot6 acute stress peak height & $1.4 \mathrm{E}-07$ & $6.3 \mathrm{E}-10$ & 1.0E-08 & $3.41 \mathrm{E}-10$ & 0.00013 \\
\hline Msn2 acute stress peak height & 0.125 & & & & \\
\hline Msn2 acclimation AUC & 0.017 & 0.0084 & & & \\
\hline $\mathrm{G} 1$ (at time of stress) $^{*}$ & 0.438 & & & & \\
\hline S-phase (at time of stress) & 0.528 & & & & \\
\hline G2 (at time of stress) & 0.233 & & & & \\
\hline M-phase (at time of stress) & 0.221 & & & & \\
\hline $\begin{array}{l}\text { Cell/colony size at experiment } \\
\text { start }\end{array}$ & 0.042 & 0.0104 & & & \\
\hline Pre-stress growth rate & $2.2 \mathrm{E}-06$ & 4.0E-07 & 2.7E-08 & & $1.59 \mathrm{E}-06$ \\
\hline $\mathrm{R}^{2}$ (explained variance) & 0.45 & 0.40 & 0.35 & 0.22 & 0.26 \\
\hline
\end{tabular}

${ }^{*}$ Cell-cycle phase included four binary parameters (i.e. dummy variables) corresponding to the four cell-cycle phases (G1, S, G2 and M phase). See the Methods section for how these were scored.

** Model 2 only included all the significant dependent variables from Model 1.

*** Model 3 excluded Msn2 acclimation AUC and Cell/colony size from Model 2 as the p-values did not pass Holm-Bonferroni correction. 